\title{
Two-way decode-and-forward cooperative systems with signal space diversity
}

\author{
Muhammad Ajmal Khan ${ }^{*}$, Raveendra K. Rao and Xianbin Wang
}

\begin{abstract}
In this paper, a novel scheme is proposed to enhance the spectral efficiency of a two-way cooperative relaying system, where two source terminals exchange information directly and via $K$ intermediate relays using the three-phase two-way decode-and-forward (DF) protocol. Signal space diversity (SSD) is incorporated into the two-way cooperative relaying system in the proposed scheme that exploits the inherent diversity in the modulation signal space by rotating and expanding the ordinary constellation. The proposed SSD-based three-phase two-way DF relaying scheme exchanges four symbols in three time slots and thus doubles the spectral efficiency as compared to the conventional three-phase two-way DF relaying system, where six time slots are required to exchange the same four symbols. This improvement in spectral efficiency is achieved without adding any extra complexity, bandwidth, or transmit power. A comprehensive analysis of the proposed scheme is carried out in this paper, and closed-form expressions for various performance metrics, including error probability, outage probability, and channel capacity, are derived. It is shown that the proposed scheme provides a diversity gain equal to one higher than the number of relays. The paper also studies different schemes for the optimization of relay position and power allocation. Finally, Monte Carlo simulations are performed which confirm the validity and accuracy of the analytical framework.
\end{abstract}

Keywords: Two-way relaying, Decode-and-forward, Signal space diversity, Spectral efficiency, Error probability, Outage probability, System optimization, Channel capacity

\section{Introduction}

Cooperative relaying has gained a lot of interest from both academic and industrial communities due to its application prospects to facilitate range-rate improvements of future wireless communication systems [1-4]. Cooperative relaying offers significant performance benefits in enabling connectivity, and increasing coverage, power saving, spectral efficiency, spatial diversity, and channel capacity $[5,6]$. A number of cooperative relaying protocols such as amplify-and-forward (AF) [7], decode-andforward (DF) [7], coded cooperation [8], compress-andforward (CF) [9], and filter-and-forward [10] are used to meet the different communication requirements [11]. In DF relaying protocol, relay decodes the information received from the source before forwarding it to the destination [12]. Moreover, a transmission node may either

\footnotetext{
*Correspondence: mkhan386@uwo.ca

Department of Electrical and Computer Engineering, The University of Western Ontario (UWO), London, ON, Canada
}

transmit or receive at a given moment in half-duplex communication [13]. In the conventional one-way cooperative relaying protocols operating in the half-duplex mode, the source broadcasts the data to the relay and the destination in the first time slot, and then the relay repeats it to the destination using its own subchannel in the next time slot [14]. This repetition-based relaying approach reduces the overall system spectral efficiency and limits the achievable data rate [15-18].

Two-way cooperative relaying establishes bidirectional connection between two source terminals to exchange independent messages with the help of relays. Operating in the half-duplex mode with DF strategy, two-way cooperative relaying has three protocols based on different channel conditions [19-21]: (i) the naive four-phase, which uses four time slots for an information exchange and is spectrally inefficient [19]; (ii) three-phase (also known as time-division broadcast channel (TDBC)) protocol $[22,23]$, which requires three time slots and is 
commonly used in two-way relaying [19]; and (iii) twophase (multiple-access broadcast channel (MABC)) protocol [24], which uses two time slots and requires network coding [25-27]. In this paper, three-phase two-way DF relaying protocol is considered due to its feature of having a direct link between both source terminals and an indirect link via intermediate relays to enhance the diversity. In the first time slot, one source terminal broadcasts its one symbol to the relay and the second source terminal. In the second time slot, the second source terminal broadcasts its one symbol to the relay and the first terminal. Then, the relay decodes the symbols received from both source terminals and broadcasts the combination of both symbols to both terminals in the third time slot. Since each source terminal perfectly knows its transmitted signal, it can cancel the self-interference term. Thus, the conventional three-phase two-way DF relaying system exchanges two symbols in three time slots [19]. To circumvent the issues due to the repetition-based relaying approach, signal space diversity (SSD) [28] is proposed to employ in the three-phase two-way DF relaying system in this paper, in order to enhance the spectral efficiency and system performance.

In SSD [28], the original symbols are rotated by a certain angle to ensure that each signal point of the rotated symbols carries enough information in its in-phase $(I)$ and quadrature $(Q)$ components in order to uniquely represent the original symbols. An $I / Q$ interleaver is employed to guarantee that each component is affected by independent channel fading. In this way, the ordinary constellation is rearranged to the expanded constellation, which contains all possible combinations of different components of signal points in the ordinary constellation. Thus, SSD extracts the inherent diversity from the modulation signal space without adding any extra bandwidth or transmit power $[29,30]$.

In this paper, we propose an SSD-based two-way DF relaying scheme using multiple relays, which provides high spectral efficiency and improved diversity. Hereafter, this proposed scheme will be referred to as SSD-based two-way cooperative system, can also called as two-way signal space cooperative system (SSC-2W). SSD has been utilized in one-way relaying systems [31-33] to improve system performance. In [31], SSD is introduced into a one-way single-relay DF cooperative system, where the source and the relay cooperate to transmit different symbols to the destination. A similar cooperative scheme with one-way single relay is analyzed in [32], where a tight closed-form upper bound on the error probability is derived. In [33], SSD is combined with space-time coding for multi-hop AF cooperative systems to improve the error performance. To the best of our knowledge, no work in the literature addresses SSD-based two-way cooperative relaying system with multiple DF relays.
In the proposed SSC-2W scheme, each of the two source terminals broadcasts its first symbol from the expanded constellation to the opposite source terminal and the relays in different time slots, i.e., the first and second time slots. Then, these symbols are decoded and the second symbols of both terminals are recovered at the relays. Based on the channel conditions between the relays and the source terminals, the best relay is selected from the group of relays that have correctly decoded the symbols. This best relay then forwards both second symbols to the source terminals in the third time slot. Thus, the proposed SSC-2W scheme exchanges four symbols in three time slots and enhances the spectral efficiency by $100 \%$ when compared with the conventional three-phase two-way DF relaying system, without adding any extra complexity, bandwidth, or transmit power.

With respect to current literature, our main contributions in this paper can be summarized in the following points: (i) a closed-form expression for the error probability is derived; (ii) an asymptotic approximation of the error probability is obtained, which clearly portrays the impact of different system parameters on the performance and confirms the diversity order achieved; (iii) the optimization of relay position under fixed power allocation (PA) and the optimization of power allocated to the source terminals and the relay under fixed relay location are investigated with the objective of minimizing the error probability; (iv) closed-form expressions for exact and asymptotic outage probabilities are derived; (v) closedform expressions for average channel capacity and an upper bound on channel capacity are obtained; and (vi) extensive Monte Carlo simulation results are obtained and compared with the analytical results, which confirms the validity and exactness of the analytical work.

The remainder of this paper is organized as follows: Section 2 describes the system model, channel model, and SSD. Section 3 explains the derivations of closedform expressions for the average symbol error probability (SEP), asymptotic SEP, and the diversity order of the system. Section 4 presents studies for the optimizations of relay position and power allocated to the source terminals and the relay. Section 5 illustrates the analysis for exact and asymptotic outage probability. Section 6 demonstrates the derivations for average channel capacity and an upper bound on channel capacity. Section 7 compares and analyzes the analytical and simulation results, and Section 8 concludes the paper.

\section{System model}

In this paper, we consider an SSD-based two-way DF relaying system, as shown in Fig. 1 . The studied system consists of two source terminals $\left(\mathrm{T}_{1}\right.$ and $\left.\mathrm{T}_{2}\right)$ and $K$ number of relays $\left(\mathrm{R}_{i}, i=1,2, \cdots, K\right)$, each with one antenna and operating in half-duplex mode. In this system, both $\mathrm{T}_{1}$ and 


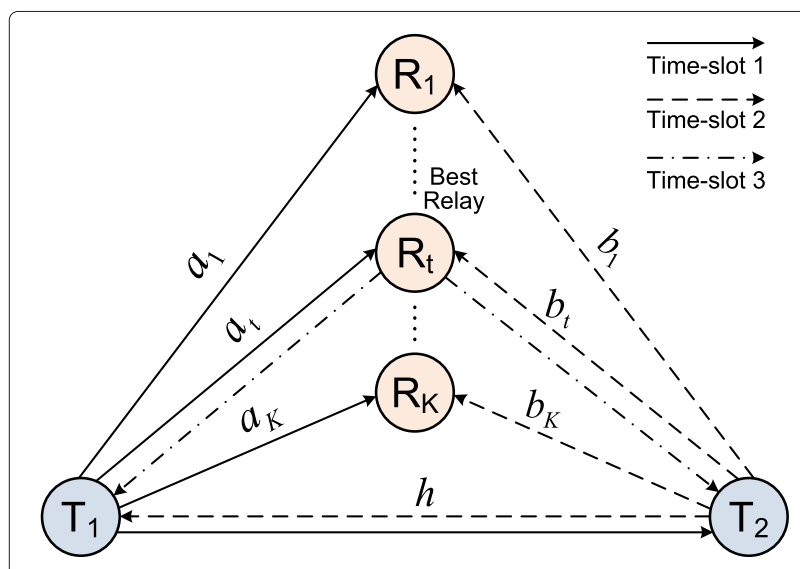

Fig. 1 System model of an SSD-based two-way cooperative system (SSC-2W) with $K$ DF relays

$\mathrm{T}_{2}$ communicate with each other directly and through $K$ relays over slow Rayleigh fading channel, whose information is available at all the receiving nodes as well as at the $K$ relays. The channel coefficients of $T_{1} \rightarrow T_{2}, R_{i} \rightarrow T_{1}$, and $\mathrm{R}_{i} \rightarrow \mathrm{T}_{2}$ links are denoted as $h, a_{i}$, and $b_{i}$ with variances $\sigma_{h}^{2}, \sigma_{a}^{2}$, and $\sigma_{b}^{2}$, respectively. The channel coefficients are assumed to be reciprocal and to remain constant during each transmission phase, and all channels are mutually independent and have no interference with each other.

The total transmit power constraint $P_{\text {tot }}$ is imposed on the exchange of every four symbols in three time slots at two source terminals and the best relay. For a fair analysis, the total transmit power $P_{\text {tot }}$ is always equal to the total power of four symbols. The symbol energies at two source terminals $\left(T_{1}\right.$ and $\left.T_{2}\right)$ and at the relays $\left(R_{i}\right)$ are denoted as $P_{T_{1}}, P_{T_{2}}$, and $P_{R}$, respectively. Zero-mean additive white Gaussian noise (AWGN) with variance $N_{0}$ is assumed over all channels. The distances of $T_{1}-T_{2}, T_{1}-R_{i}$, and $\mathrm{T}_{2}-\mathrm{R}_{i}$ links are denoted as $d_{T_{1} T_{2}}, d_{T_{1} R}$, and $d_{T_{2} R}$, respectively, and their geometric gains are represented as $\lambda_{T_{1} R}=\left(d_{T_{1} T_{2}} / d_{T_{1} R}\right)^{v}$ and $\lambda_{T_{2} R}=\left(d_{T_{1} T_{2}} / d_{T_{2} R}\right)^{v}$, respectively, where $v$ is the path loss exponent. The instantaneous signal-to-noise ratios (SNRs) of $\mathrm{T}_{1} \rightarrow \mathrm{T}_{2}, \mathrm{~T}_{2} \rightarrow$ $\mathrm{T}_{1}, \mathrm{~T}_{1} \rightarrow \mathrm{R}_{i}, \mathrm{~T}_{2} \rightarrow \mathrm{R}_{i}, \mathrm{R}_{i} \rightarrow \mathrm{T}_{1}$, and $\mathrm{R}_{i} \rightarrow \mathrm{T}_{2}$ links are denoted as $\gamma_{h_{1}}=|h|^{2} P_{T_{1}} / N_{0}, \gamma_{h_{2}}=|h|^{2} P_{T_{2}} / N_{0}$, $\gamma_{s a_{i}}=\left|a_{i}\right|^{2} \lambda_{T_{1} R} P_{T_{1}} / N_{0}, \gamma_{s b_{i}}=\left|b_{i}\right|^{2} \lambda_{T_{2} R} P_{T_{2}} / N_{0}, \gamma_{a_{i}}=$ $\left|a_{i}\right|^{2} \lambda_{T_{1} R} P_{R} / N_{0}$, and $\gamma_{b_{i}}=\left|b_{i}\right|^{2} \lambda_{T_{2} R} P_{R} / N_{0}$, while average SNRs are $\bar{\gamma}_{h_{1}}=\sigma_{h}^{2} P_{T_{1}} / N_{0}, \bar{\gamma}_{h_{2}}=\sigma_{h}^{2} P_{T_{2}} / N_{0}$, $\bar{\gamma}_{s a}=\sigma_{a}^{2} \lambda_{T_{1} R} P_{T_{1}} / N_{0}, \bar{\gamma}_{s b}=\sigma_{b}^{2} \lambda_{T_{2} R} P_{T_{2}} / N_{0}, \bar{\gamma}_{a}=$ $\sigma_{a}^{2} \lambda_{T_{1} R} P_{R} / N_{0}$, and $\bar{\gamma}_{b}=\sigma_{b}^{2} \lambda_{T_{2} R} P_{R} / N_{0}$, respectively.

In the studied system, SSD is applied at the source terminals and the relays. Figure 2 shows the constellation rotation and expansion in the SSD. First, each $m=$ $\log _{2}(M)$ information bits at each source terminal are grouped and mapped to some ordinary constellation using a digital modulation scheme such as $M$-ary quadrature amplitude modulation ( $M$-QAM) or $M$-ary phase-shift keying (M-PSK).

Let $\mathbf{s}=\left(s_{1}, s_{2}\right)$ be the two original signal points from the ordinary constellation $\Phi$ (e.g., 4-QAM, or QPSK), i.e., $s_{1}, s_{2} \in \Phi$. The original complex symbols can be denoted as $s_{1}=\Re\left\{s_{1}\right\}+j \mathfrak{s}\left\{s_{1}\right\}$ and $s_{2}=\Re\left\{s_{2}\right\}+j \mathfrak{s}\left\{s_{2}\right\}$, where $j=\sqrt{-1}$, and $\Re\{\cdot\}$ and $\Im\{\cdot\}$ are $I$ and $Q$ components of the symbols, respectively. Then, $s_{1}$ and $s_{2}$ are rotated by the angle $\theta$, i.e., $x_{1}=s_{1} e^{j \theta}$ and $x_{2}=s_{2} e^{j \theta}$. The rotated symbols, $\mathbf{x}=\left(x_{1}, x_{2}\right)$, correspond to a rotated constellation $\Phi_{r}$, which is generated by applying a transformation $\Theta$ to the ordinary constellation as

$$
\Theta=\left[\begin{array}{cc}
\cos \theta & -\sin \theta \\
\sin \theta & \cos \theta
\end{array}\right] .
$$

The rotation angle $\theta$ is chosen carefully to ensure that each signal point in the rotated constellation carries enough information in one component, either $I$ or $Q$, and uniquely represents the original signal [28, 31]. A list of $\theta$ for various modulation schemes can be found in $[34,35]$, such as the rotation angle $\theta$ of $26.6^{\circ}, 14.0^{\circ}$, and $7.1^{\circ}$, is chosen for 4-, 16-, and 64-QAM constellations, respectively. The new SSD symbols, $\mathbf{z}=\left(z_{1}, z_{2}\right)$, for transmission are formed by interleaving the $Q$ components of the rotated symbols, $x_{1}$ and $x_{2}$, and are given by

$$
\begin{aligned}
& z_{1}=\Re\left\{x_{1}\right\}+j \mathfrak{s}\left\{x_{2}\right\}, \\
& z_{2}=\Re\left\{x_{2}\right\}+j \mathfrak{s}\left\{x_{1}\right\} .
\end{aligned}
$$

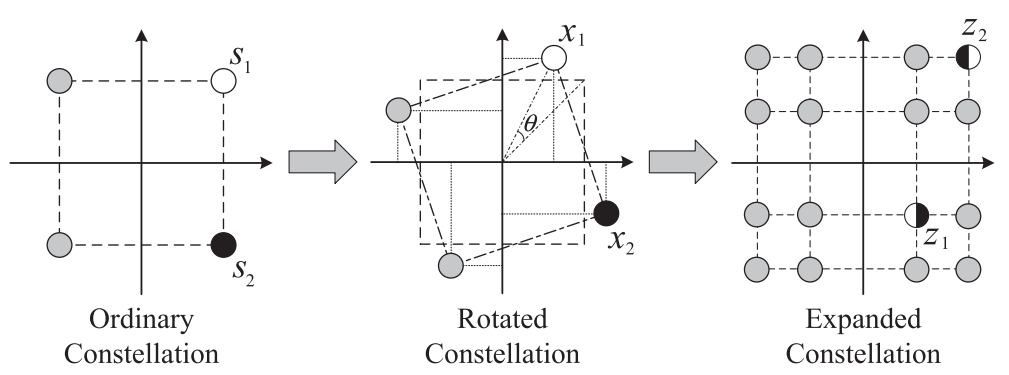

Fig. 2 Ordinary to expanded constellation in SSD 
The SSD symbols, $z_{1}$ and $z_{2}$, for transmission belong to an expanded constellation $\Upsilon$, i.e $\Upsilon=\Re\left\{\Phi_{r}\right\} \times \Im\left\{\Phi_{r}\right\}$, where $\times$ is the Cartesian product of two sets. It is significant to mention that each member of $\Upsilon$ consists of two components, i.e., a real component from a member of $\Phi_{r}$ and an imaginary component from another member of $\Phi_{r}$. Moreover, each of the component identifies a specific member of $\Phi_{r}$. Thus, decoding a member of $\Upsilon$ leads to decoding two different members of $\Phi_{r}$ (i.e., $x_{1}$ and $x_{2}$ ). It is important to highlight that the expanded constellation, resulting from component interleaving and ordinary constellation rotation, does not convert a low order constellation to higher order. In addition, the expanded constellation maintains the same number of bits per signal point as in the ordinary constellation.

To this end, we explain the use of SSD in the three-phase two-way DF relaying system. Firstly, $\mathrm{T}_{1}$ prepares two SSD symbols, $z_{T_{1}, 1}$ and $z_{T_{1}, 2}$, by component interleaving of its two original rotated symbols, $x_{T_{1}, 1}$ and $x_{T_{1}, 2}$, as

$$
\begin{aligned}
& z_{T_{1}, 1}=\Re\left\{x_{T_{1}, 1}\right\}+j \Im\left\{x_{T_{1}, 2}\right\}, \\
& z_{T_{1}, 2}=\Re\left\{x_{T_{1}, 2}\right\}+j \Im\left\{x_{T_{1}, 1}\right\} .
\end{aligned}
$$

In the first time slot, $\mathrm{T}_{1}$ broadcasts only one of the two SSD symbols (i.e. $z_{T_{1}, 1}$ ). Thus, the received signal at $T_{2}$ and the $i$ th relay can be written as

$$
\begin{aligned}
y_{T_{1} T_{2}} & =\sqrt{P_{T_{1}}} h z_{T_{1}, 1}+w_{T_{2}, 1}, \\
y_{T_{1} R_{i}} & =\sqrt{P_{T_{1}} \lambda_{T_{1} R}} a_{i} z_{T_{1}, 1}+w_{T_{1}, R_{i}},
\end{aligned}
$$

where $w_{T_{2}, 1}$ and $w_{T_{1}, R_{i}}$ denote zero-mean AWGN with a variance of $N_{0}$ at $\mathrm{T}_{2}$ and the $i$ th relay, respectively. The two original rotated symbols, $x_{T_{1}, 1}$ and $x_{T_{1}, 2}$, are detected using the transmitted SSD symbol, $z_{T_{1}, 1}=\Re\left\{x_{T_{1}, 1}\right\}+$ $j \Im\left\{x_{T_{1}, 2}\right\}$, at the $i$ th relay as

$\hat{x}_{T_{1}, 1}=\left.\left.\arg \min _{x \in \Phi_{r}}\left|\Re\left\{a_{i}^{*} y_{T_{1} R_{i}}\right\}-\sqrt{P_{T_{1}} \lambda_{T_{1} R}}\right| a_{i}\right|^{2} \Re\left\{x_{T_{1}, 1}\right\}\right|^{2}$,

$\hat{x}_{T_{1}, 2}=\left.\left.\arg \min _{x \in \Phi_{r}}\left|\mathfrak{\Im}\left\{a_{i}^{*} y_{T_{1} R_{i}}\right\}-\sqrt{P_{T_{1}} \lambda_{T_{1} R}}\right| a_{i}\right|^{2} \mathfrak{\Im}\left\{x_{T_{1}, 2}\right\}\right|^{2}$,

where $*$ denotes complex conjugation.

Secondly, $\mathrm{T}_{2}$ prepares two SSD symbols, $z_{T_{2}, 1}$ and $z_{T_{2}, 2}$, by component interleaving of its two original rotated symbols, $x_{T_{2}, 1}$ and $x_{T_{2}, 2}$, as

$$
\begin{aligned}
& z_{T_{2}, 1}=\Re\left\{x_{T_{2}, 1}\right\}+j \Im\left\{x_{T_{2}, 2}\right\}, \\
& z_{T_{2}, 2}=\Re\left\{x_{T_{2}, 2}\right\}+j \Im\left\{x_{T_{2}, 1}\right\} .
\end{aligned}
$$

Similarly, in the second time slot, $\mathrm{T}_{2}$ broadcasts only one of the two SSD symbols (i.e., $z_{T_{2}, 1}$ ), which is received at $\mathrm{T}_{1}$ and the $i$ th relay as

$$
\begin{aligned}
y_{T_{2} T_{1}} & =\sqrt{P_{T_{2}}} h z_{T_{2}, 1}+w_{T_{1}, 1}, \\
y_{T_{2} R_{i}} & =\sqrt{P_{T_{2}} \lambda_{T_{2} R}} b_{i} z_{T_{2}, 1}+w_{T_{2}, R_{i}},
\end{aligned}
$$

where $w_{T_{1}, 1}$ and $w_{T_{2}, R_{i}}$ denote zero-mean AWGN with a variance of $N_{0}$ at $\mathrm{T}_{1}$ and the $i$ th relay, respectively. Then, the two original rotated symbols, $x_{T_{2}, 1}$ and $x_{T_{2}, 2}$, are detected using the transmitted SSD symbol, $z_{T_{2}, 1}$, at the $i$ th relay as

$$
\begin{aligned}
& \hat{x}_{T_{2}, 1}=\left.\left.\arg \min _{x \in \Phi_{r}}\left|\Re\left\{b_{i}^{*} y_{T_{2} R_{i}}\right\}-\sqrt{P_{T_{2}} \lambda_{T_{2} R}}\right| b_{i}\right|^{2} \Re\left\{x_{T_{2}, 1}\right\}\right|^{2}, \\
& \hat{x}_{T_{2}, 2}=\left.\left.\arg \min _{x \in \Phi_{r}}\left|\Im\left\{b_{i}^{*} y_{T_{2} R_{i}}\right\}-\sqrt{P_{T_{2}} \lambda_{T_{2} R}}\right| b_{i}\right|^{2} \Im\left\{x_{T_{2}, 2}\right\}\right|^{2} .
\end{aligned}
$$

Thanks to the SSD technique that an SSD symbol from a terminal received at the relay consists of two components to represent two different original symbols. If the relay successfully decodes an SSD symbol, $z_{T_{1}, 1}$, from $T_{1}$, it can recover both symbols, $x_{T_{1}, 1}$ and $x_{T_{1}, 2}$, correctly due to the SSD technique. Similarly, the relay can recover $x_{T_{2}, 1}$ and $x_{T_{2}, 2}$ correctly upon successful decoding of $z_{T_{2}, 1}$ from $T_{2}$. Otherwise, the failure in decoding is conveyed through a reliable feedback channel to the terminals. These assumptions are based on ARQ and CRC protection features which are part of the advanced wireless standards such as IEEE 802.16 [36]. Let $\mathcal{D}$ be the set of relays that have correctly decoded the messages in the first and second time slots, and $|\mathcal{D}|$ its cardinality. Let $\mathrm{R}_{t}$ denotes the best relay among all relays that correctly decode both source messages and have good channel conditions to both source terminals. Thus, the relay selection criteria, based on $\gamma_{a_{i}}$ and $\gamma_{b_{i}}$, can be expressed as

$$
\mathrm{R}_{t}=\arg \max _{i \in \mathcal{D}}\left\{\min \left(\gamma_{a_{i}}, \gamma_{b_{i}}\right)\right\} .
$$

The best relay $\left(\mathrm{R}_{t}\right)$ forwards the combined signal of $z_{T_{1}, 2}$ and $z_{T_{2}, 2}$ to the source terminals in the third time slot. Thus, the received signals at the source terminals can be written as

$$
\begin{aligned}
& y_{R_{t} T_{1}}=\sqrt{P_{R} \lambda_{T_{1} R}} a_{t} z_{T_{1}, 2}+\sqrt{P_{R} \lambda_{T_{1} R}} a_{t} z_{T_{2}, 2}+w_{1,2}, \\
& y_{R_{t} T_{2}}=\sqrt{P_{R} \lambda_{T_{2} R}} b_{t} z_{T_{1}, 2}+\sqrt{P_{R} \lambda_{T_{2} R}} b_{t} z_{T_{2}, 2}+w_{2,2},
\end{aligned}
$$

where $w_{1,2}$ and $w_{2,2}$ denote zero-mean AWGN with a variance of $N_{0}$ at $T_{1}$ and $T_{2}$, respectively. Since each terminal perfectly knows its transmitted signal, it can cancel the self-interference term. Thus, the resulting signals at $T_{1}$ and $\mathrm{T}_{2}$ can be written as

$$
\begin{aligned}
& y_{R_{t} T_{1}}=\sqrt{P_{R} \lambda_{T_{1} R}} a_{t} z_{T_{2}, 2}+w_{1,2}, \\
& y_{R_{t} T_{2}}=\sqrt{P_{R} \lambda_{T_{2} R}} b_{t} z_{T_{1}, 2}+w_{2,2} .
\end{aligned}
$$


Let $\mathbf{r}_{T_{1}}=\left(r_{T_{1}, 1}, r_{T_{1}, 2}\right)$ be the signal after component de-interleaver at $T_{2}$, transmitted by $T_{1}$, which can be expressed as

$$
\begin{aligned}
& r_{T_{1}, 1}=\Re\left\{h^{*} y_{T_{1} T_{2}}\right\}+j \Im\left\{b_{t}^{*} y_{R_{t} T_{2}}\right\}, \\
& r_{T_{1}, 2}=\Re\left\{b_{t}^{*} y_{R_{t} T_{2}}\right\}+j \Im\left\{h^{*} y_{T_{1} T_{2}}\right\} .
\end{aligned}
$$

The maximum likelihood detection is applied at $T_{2}$ in order to detect the message transmitted by $T_{1}$ using

$$
\begin{aligned}
\hat{x}_{T_{1}, 1}= & \arg \min _{x \in \Phi_{r}}\left[\left.\left.\left|\Re\left\{r_{T_{1}, 1}\right\}-\sqrt{P_{T_{1}}}\right| h\right|^{2} \Re\left\{x_{T_{1}, 1}\right\}\right|^{2}\right. \\
& \left.+\left.\left.\left|\Im\left\{r_{T_{1}, 1}\right\}-\sqrt{P_{R} \lambda_{T_{2} R}}\right| b_{t}\right|^{2} \Im\left\{x_{T_{1}, 1}\right\}\right|^{2}\right], \quad \text { (23) } \\
\hat{x}_{T_{1}, 2}= & \arg \min _{x \in \Phi_{r}}\left[\left.\left.\left|\Re\left\{r_{T_{1}, 2}\right\}-\sqrt{P_{R} \lambda_{T_{2} R}}\right| b_{t}\right|^{2} \Re\left\{x_{T_{1}, 2}\right\}\right|^{2}\right. \\
& \left.+\left.\left.\left|\Im\left\{r_{T_{1}, 2}\right\}-\sqrt{P_{T_{1}}}\right| h\right|^{2} \Im\left\{x_{T_{1}, 2}\right\}\right|^{2}\right] .
\end{aligned}
$$

Similarly, the signal at $\mathrm{T}_{1}, \mathbf{r}_{T_{2}}=\left(r_{T_{2}, 1}, r_{T_{2}, 2}\right)$, after component de-interleaver can be expressed as

$$
\begin{aligned}
& r_{T_{2}, 1}=\Re\left\{h^{*} y_{T_{2} T_{1}}\right\}+j \Im\left\{a_{t}^{*} y_{R_{t} T_{1}}\right\}, \\
& r_{T_{2}, 2}=\Re\left\{a_{t}^{*} y_{R_{t} T_{1}}\right\}+j \Im\left\{h^{*} y_{T_{2} T_{1}}\right\} .
\end{aligned}
$$

The message at $T_{1}$, transmitted by $T_{2}$, is detected as

$$
\begin{aligned}
\hat{x}_{T_{2}, 1}= & \arg \min _{x \in \Phi_{r}}\left[\left.\left.\left|\Re\left\{r_{T_{2}, 1}\right\}-\sqrt{P_{T_{2}}}\right| h\right|^{2} \Re\left\{x_{T_{2}, 1}\right\}\right|^{2}\right. \\
& \left.+\left.\left.\left|\Im\left\{r_{T_{2}, 1}\right\}-\sqrt{P_{R} \lambda_{T_{1} R}}\right| a_{t}\right|^{2} \Im\left\{x_{T_{2}, 1}\right\}\right|^{2}\right], \quad \text { (27) } \\
\hat{x}_{T_{2}, 2}= & \arg \min _{x \in \Phi_{r}}\left[\left.\left.\left|\Re\left\{r_{T_{2}, 2}\right\}-\sqrt{P_{R} \lambda_{T_{1} R}}\right| a_{t}\right|^{2} \Re\left\{x_{T_{2}, 2}\right\}\right|^{2}\right. \\
& \left.+\left.\left.\left|\Im\left\{r_{T_{2}, 2}\right\}-\sqrt{P_{T_{2}}}\right| h\right|^{2} \Im\left\{x_{T_{2}, 2}\right\}\right|^{2}\right] .
\end{aligned}
$$

It is important to note that the proposed SSC-2W scheme exchanges four symbols in three time slots. On the other hand, the conventional three-phase two-way DF relaying system [19] requires three time slots to exchange two symbols, thus requiring six time slots for four symbols. In addition, if one of the two transmitted SSD symbols are not received correctly at the opposite terminal in the proposed SSC-2W scheme, it will still be able to recover both symbols from one SSD symbol due to the SSD technique. Therefore, the proposed SSC-2W scheme increases the data rate, spectral efficiency, and diversity when compared with the conventional three-phase twoway DF relaying system without any additional bandwidth or transmit power.

\section{Error probability analysis}

In this section, we derive a closed-form expression for the end-to-end error probability of the proposed SSC2W scheme over Rayleigh fading channel. Moreover, we analyze asymptotic approximation of the error probability and diversity gain of the system.

\subsection{Average error probability}

The average SEP of the system depends on the probability that the relays detect the signal transmitted from source terminals with or without an error, and the probability of erroneous transmission from one source terminal to the other. In addition, when a set of relays is available that have correctly received signals from source terminals, the average SEP of the system depends on the cooperative links between the best relay and the source terminals. Due to two-way communication, we first focus our analysis at terminal $T_{2}$ for the signal transmitted by terminal $T_{1}$. We define $\operatorname{Pr}\{|\mathcal{D}|=0\}$ as the probability that no relay is able to correctly detect the signals transmitted by source terminals, and $\operatorname{Pr}\{|\mathcal{D}|=k\}$ as the probability that $k$ relays detect source signals correctly. Moreover, $\mathcal{P}_{\text {sym }}^{T_{1} \text {,cop }}(e \mid k)$ is the conditional error probability of the cooperative link between $T_{1} \rightarrow T_{2}$ and $R_{t} \rightarrow T_{2}$ when $k$ relays detect both source signals correctly. Furthermore, $\mathcal{P}_{\text {sym }}^{T_{1} T_{2}}(e)$ and $\mathcal{P}_{\text {sym }}^{T_{2} T_{1}}(e)$ are the error probabilities of a signal transmitted from $T_{1}$ to $T_{2}$ and $T_{2}$ to $T_{1}$, respectively. Thus, the average SEP of the signal transmitted by $T_{1}$ can be written as

$$
\mathcal{P}_{\text {sym }}^{\mathrm{T}_{1}}(e)=\operatorname{Pr}\{|\mathcal{D}|=0\} \mathcal{P}_{\text {sym }}^{T_{1} T_{2}}(e)+\sum_{k=1}^{K} \operatorname{Pr}\{|\mathcal{D}|=k\} \mathcal{P}_{\text {sym }}^{T_{1}, \text { coop }}(e \mid k),
$$

where

$$
\begin{aligned}
& \operatorname{Pr}\{|\mathcal{D}|=0\}=\left[\mathcal{P}_{\text {off }}\right]^{K}, \\
& \operatorname{Pr}\{|\mathcal{D}|=k\}=\left(\begin{array}{l}
K \\
k
\end{array}\right)\left[\mathcal{P}_{\text {off }}\right]^{K-k}\left[1-\mathcal{P}_{\text {off }}\right]^{k},
\end{aligned}
$$

where $\mathcal{P}_{\text {off }}$ denotes the probability that a relay fails to detect both source signals correctly and remains silent during the third time slot. If $\mathcal{P}_{\text {sym }}^{T_{1} R_{i}}(e)$ and $\mathcal{P}_{\text {sym }}^{T_{2} R_{i}}(e)$ denote the error probabilities at the $i$ th relay for signal transmitted by $T_{1}$ and $T_{2}$, respectively, then $\mathcal{P}_{\text {off }}$ can be written as

$$
\mathcal{P}_{\text {off }}=1-\left[1-\mathcal{P}_{\text {sym }}^{T_{1} R_{i}}(e)\right]\left[1-\mathcal{P}_{\text {sym }}^{T_{2} R_{i}}(e)\right] .
$$

The average SEP of $T_{1}$ can then be expressed as

$$
\begin{aligned}
\mathcal{P}_{\text {sym }}^{T_{1}}(e)= & {\left[\mathcal{P}_{\text {off }}\right]^{K} \mathcal{P}_{\text {sym }}^{T_{1} T_{2}}(e)+\sum_{k=1}^{K}\left(\begin{array}{c}
K \\
k
\end{array}\right) } \\
& \times\left[\mathcal{P}_{\text {off }}\right]^{K-k}\left[1-\mathcal{P}_{\text {off }}\right]^{k} \mathcal{P}_{\text {sym }}^{T_{1}, \text { coop }}(e \mid k) .
\end{aligned}
$$

In what follows, we derive the expressions for each of these probabilities.

The conditional SEP for a given $\gamma_{s a_{i}}$ of the $\mathrm{T}_{1} \rightarrow \mathrm{R}_{i}$ link, $\mathcal{P}_{\text {sym }}^{T_{1} R_{i}}\left(e \mid \gamma_{s a_{i}}\right)$, can be tightly upper bounded as

$$
\mathcal{P}_{\text {sym }}^{T_{1} R_{i}}\left(e \mid \gamma_{s a_{i}}\right) \leq \alpha_{z} Q\left(\sqrt{\beta_{z} \gamma_{s a_{i}}}\right),
$$


where $Q(u)=\frac{1}{\sqrt{2 \pi}} \int_{u}^{\infty} e^{-t^{2} / 2} d t$ is the Gaussian Qfunction ([37] 26.2.3), $\alpha_{z}$ and $\beta_{z}$ are determined by the type of the expanded constellation and its size $|\Upsilon|$. For example, for $M$-QAM, $\alpha_{z}=4$ and $\beta_{z}=3 /(|\Upsilon|-1)$; and for $M$-PSK, $\alpha_{z}=2$ and $\beta_{z}=2 \sin ^{2}(\pi /|\Upsilon|)$ [38]. Therefore, $\mathcal{P}_{\text {sym }}^{T_{1} R_{i}}(e)$ over Rayleigh fading channel can be written as

$$
\mathcal{P}_{\text {sym }}^{T_{1} R_{i}}(e) \leq \int_{0}^{\infty} \alpha_{z} Q\left(\sqrt{\beta_{z} \gamma_{s a_{i}}}\right) f_{\gamma_{s a_{i}}}\left(\gamma_{s a_{i}}\right) d \gamma_{s a_{i}},
$$

where $f_{\gamma_{s a_{i}}}\left(\gamma_{s a_{i}}\right)=\frac{1}{\bar{\gamma}_{s a}} e^{-\gamma_{s a_{i}} / \bar{\gamma}_{s a}}$ is the PDF of the SNR $\gamma_{s a_{i}}$ [39]. We formulate the following expression using [40]

$$
\int_{0}^{\infty} A Q(\sqrt{B t}) \frac{1}{C} e^{-t / C} d t=\frac{1}{2} A\left(1-\sqrt{\frac{B C}{B C+2}}\right) .
$$

Solving the integration in Eq. (35) using Eq. (36), $\mathcal{P}_{\text {sym }}^{T_{1} R_{i}}(e)$ can be written as

$$
\mathcal{P}_{\text {sym }}^{T_{1} R_{i}}(e) \leq \frac{1}{2} \alpha_{z}\left(1-\sqrt{\frac{\beta_{z} \bar{\gamma}_{s a}}{\beta_{z} \bar{\gamma}_{s a}+2}}\right) .
$$

Similarly, the SEP at the $i$ th relay for a signal transmitted by $\mathrm{T}_{2}$ over Rayleigh fading channel can be expressed as

$$
\mathcal{P}_{\mathrm{sym}}^{T_{2} R_{i}}(e) \leq \frac{1}{2} \alpha_{z}\left(1-\sqrt{\frac{\beta_{z} \bar{\gamma}_{s b}}{\beta_{z} \bar{\gamma}_{s b}+2}}\right),
$$

and the SEP at $T_{2}$ for a signal transmitted by $T_{1}$ over Rayleigh fading channel can be written as

$$
\mathcal{P}_{\text {sym }}^{T_{1} T_{2}}(e) \leq \frac{1}{2} \alpha_{z}\left(1-\sqrt{\frac{\beta_{z} \bar{\gamma}_{h_{1}}}{\beta_{z} \bar{\gamma}_{h_{1}}+2}}\right) .
$$

The SEP of the cooperative link between $T_{1} \rightarrow T_{2}$ and $\mathrm{R}_{t} \rightarrow \mathrm{T}_{2}$ depends on the total SNR of the cooperative link, $\gamma_{e_{1}}=\gamma_{h_{1}}+\gamma_{b_{t}}$, where $\gamma_{b_{t}}$ is the SNR of the best relay chosen under the relay selection criterion, given by Eq. (16). Thus, the SEP of the cooperative link for given SNRs $\gamma_{a_{i}}$, $\gamma_{b_{i}}, \gamma_{h_{1}}$ and $k$ active relays can be represented as [38]

$$
\mathcal{P}_{\text {sym }}^{T_{1} \text {,coop }}\left(e \mid k, \gamma_{h_{1}}, \gamma_{a_{i}}, \gamma_{b_{i}}\right)=\alpha_{x} Q\left(\sqrt{\beta_{x} \gamma_{e_{1}}}\right),
$$

where $\alpha_{x}=2\left(\left|\Phi_{r}\right|-1\right) /\left|\Phi_{r}\right|, \beta_{x}=3 /\left(\left|\Phi_{r}\right|^{2}-1\right)$ [38]. Then, $\mathcal{P}_{\text {sym }}^{T_{1}, \text { coop }}(e \mid k)$ over the fading channel can be written as

$$
\mathcal{P}_{\text {sym }}^{T_{1}, \text { coop }}(e \mid k)=\int_{0}^{\infty} \alpha_{x} Q\left(\sqrt{\beta_{x} \gamma_{e_{1}}}\right) f_{\gamma_{e_{1}}}\left(\gamma_{e_{1}}\right) d \gamma_{e_{1}},
$$

where $f_{\gamma_{e_{1}}}\left(\gamma_{e_{1}}\right)$ is the PDF of the SNR $\gamma_{e_{1}}$. To evaluate $f_{\gamma_{e_{1}}}\left(\gamma_{e_{1}}\right)$, PDF of $\gamma_{b_{t}}$ is derived first with the help of the PDFs of $\gamma_{a_{i}}$ and $\gamma_{b_{i}}$, that are $f_{\gamma_{a_{i}}}\left(\gamma_{a_{i}}\right)=\frac{1}{\bar{\gamma}_{a}} e^{-\gamma_{a_{i}} / \bar{\gamma}_{a}}$ and $f_{\gamma_{b_{i}}}\left(\gamma_{b_{i}}\right)=\frac{1}{\bar{\gamma}_{b}} e^{-\gamma_{b_{i}} / \bar{\gamma}_{b}}$, respectively. Thus, using ([41] Appendix A), $f_{\gamma_{b_{t}}}(\gamma)$ can be expressed as

$$
\begin{aligned}
f_{\gamma_{b}}(\gamma)= & \sum_{k=1}^{|\mathcal{D}|}\left(\begin{array}{c}
|\mathcal{D}| \\
k
\end{array}\right) \frac{(-1)^{k-1}}{\bar{\gamma}_{a}} \frac{k \bar{\gamma}_{m}}{k \bar{\gamma}_{b}-\bar{\gamma}_{m}}\left(e^{-\gamma / \bar{\gamma}_{b}}-e^{-k \gamma / \bar{\gamma}_{m}}\right) \\
& +\sum_{k=1}^{|\mathcal{D}|}\left(\begin{array}{c}
|\mathcal{D}| \\
k
\end{array}\right) \frac{(-1)^{k-1}}{\bar{\gamma}_{b}} k e^{-k \gamma / \bar{\gamma}_{m}},
\end{aligned}
$$

where $\bar{\gamma}_{m}=\bar{\gamma}_{a} \bar{\gamma}_{b} /\left(\bar{\gamma}_{a}+\bar{\gamma}_{b}\right) \cdot f_{\gamma_{e_{1}}}(\gamma)$ is evaluated by convolving $f_{\gamma_{h_{1}}}(\gamma)=\frac{1}{\bar{\gamma}_{h_{1}}} e^{-\gamma / \bar{\gamma}_{h_{1}}}$ and $f_{\gamma_{b_{t}}}(\gamma)$ from Eq. (42); thus, $f_{\gamma_{e_{1}}}(\gamma)$ can be expressed as

$$
\begin{aligned}
f_{\gamma_{e_{1}}}(\gamma)= & \sum_{k=1}^{|\mathcal{D}|}\left(\begin{array}{c}
|\mathcal{D}| \\
k
\end{array}\right) \frac{(-1)^{k-1}}{\bar{\gamma}_{a}} \frac{k \bar{\gamma}_{m}}{k \bar{\gamma}_{b}-\bar{\gamma}_{m}}\left[\frac{\bar{\gamma}_{b}}{\bar{\gamma}_{h_{1}}-\bar{\gamma}_{b}}\left(e^{-\gamma / \bar{\gamma}_{h_{1}}}-e^{-\gamma / \bar{\gamma}_{b}}\right)\right. \\
& \left.-\frac{\bar{\gamma}_{m}}{k \bar{\gamma}_{h_{1}}-\bar{\gamma}_{m}}\left(e^{-\gamma / \bar{\gamma}_{h_{1}}}-e^{-k \gamma / \bar{\gamma}_{m}}\right)\right] \\
& +\sum_{k=1}^{|\mathcal{D}|}\left(\begin{array}{c}
|\mathcal{D}| \\
k
\end{array}\right) \frac{(-1)^{k-1}}{\bar{\gamma}_{b}} \frac{k \bar{\gamma}_{m}}{k \bar{\gamma}_{h_{1}}-\bar{\gamma}_{m}}\left(e^{-\gamma / \bar{\gamma}_{h_{1}}}-e^{-k \gamma / \bar{\gamma}_{m}}\right) .
\end{aligned}
$$

Substituting Eq. (43) into Eq. (41) and solving the integration using Eq. (36), $\mathcal{P}_{\text {sym }}^{T_{1} \text {,coop }}(e \mid k)$ can be expressed as

$$
\begin{aligned}
& \mathcal{P}_{\text {sym }}^{T_{1}, \text { coop }}(e \mid k)=\frac{1}{2} \alpha_{x} \sum_{i=1}^{k}\left(\begin{array}{c}
k \\
i
\end{array}\right) \frac{(-1)^{i-1}}{\bar{\gamma}_{a}} \frac{i \bar{\gamma}_{m}}{i \bar{\gamma}_{b}-\bar{\gamma}_{m}} \times \\
& {\left[\frac{\bar{\gamma}_{b}}{\bar{\gamma}_{h_{1}}-\bar{\gamma}_{b}}\left\{\bar{\gamma}_{h_{1}}\left(1-\sqrt{\frac{\beta_{x} \bar{\gamma}_{h_{1}}}{\beta_{x} \bar{\gamma}_{h_{1}}+2}}\right)-\bar{\gamma}_{b}\left(1-\sqrt{\frac{\beta_{x} \bar{\gamma}_{b}}{\beta_{x} \bar{\gamma}_{b}+2}}\right)\right\}\right.} \\
& \left.-\frac{\bar{\gamma}_{m}}{\bar{\gamma}_{h_{1}}-\bar{\gamma}_{m}}\left\{\bar{\gamma}_{h_{1}}\left(1-\sqrt{\frac{\beta_{x} \bar{\gamma}_{h_{1}}}{\beta_{x} \bar{\gamma}_{h_{1}}+2}}\right)-\frac{\bar{\gamma}_{m}}{i}\left(1-\sqrt{\frac{\beta_{x} \bar{\gamma}_{m}}{\beta_{x} \bar{\gamma}_{m}+2 i}}\right)\right\}\right] \\
& +\frac{1}{2} \alpha_{x} \sum_{i=1}^{k}\left(\begin{array}{c}
k \\
i
\end{array}\right) \frac{(-1)^{i-1}}{\bar{\gamma}_{b}} \frac{i \bar{\gamma}_{m}}{i \bar{\gamma}_{h_{1}}-\bar{\gamma}_{m}}\left[\bar{\gamma}_{h_{1}}\left(1-\sqrt{\frac{\beta_{x} \bar{\gamma}_{h_{1}}}{\beta_{x} \bar{\gamma}_{h_{1}}+2}}\right)\right. \\
& \left.\quad-\frac{\bar{\gamma}_{m}}{i}\left(1-\sqrt{\frac{\beta_{x} \bar{\gamma}_{m}}{\beta_{x} \bar{\gamma}_{m}+2 i}}\right)\right] .
\end{aligned}
$$

Finally, substituting Eqs. (37), (38), (39), and (44) into Eq. (33), a closed-form expression for the average SEP at the terminal $T_{2}$ for the signal transmitted by $T_{1}$ can be easily obtained. It is noted that a similar expression can be obtained for the average SEP at the terminal $T_{1}$ for the signal transmitted by $T_{2}, \mathcal{P}_{\text {sym }}^{T_{2}}(e)$, by following the same procedure or simply replacing $\bar{\gamma}_{h_{1}}$ with $\bar{\gamma}_{h_{2}}$, and by interchanging $\bar{\gamma}_{b}$ and $\bar{\gamma}_{a}$ in the expression for $\mathcal{P}_{\text {sym }}^{T_{1}}(e)$. 
Thus, the end-to-end average SEP of the proposed SSC$2 \mathrm{~W}$ scheme can be obtained using the following relation

$$
\mathcal{P}_{\text {sym }}^{e 2 e}(e)=\frac{\mathcal{P}_{\text {sym }}^{T_{1}}(e)+\mathcal{P}_{\text {sym }}^{T_{2}}(e)}{2} .
$$

\subsection{Asymptotic error probability}

Although the derived average SEP expression of the system is very useful in evaluating error performance of the system, it is not straightforward to use this expression to quantify the diversity gain and the effect of system parameters. Consequently, we derive simple asymptotic SEP expression, which is of special interest in moderate to high SNR region. The asymptotic SEP at the terminal $T_{2}$ for the signal transmitted by $T_{1}$ can be expressed as

$\mathcal{P}_{\text {sym }}^{T_{1}}(e) \simeq\left[\mathcal{P}_{\text {off }}\right]^{K} \mathcal{P}_{\text {sym }}^{T_{1} T_{2}}(e)+\sum_{k=1}^{K}\left(\begin{array}{c}K \\ k\end{array}\right)\left[\mathcal{P}_{\text {off }}\right]^{K-k} \mathcal{P}_{\text {sym }}^{T_{1}, \text { coop }}(e \mid k)$.

For high SNR, $\mathcal{P}_{\text {off }}$ from Eq. (32) can be approximated as

$$
\mathcal{P}_{\text {off }} \approx \mathcal{P}_{\text {sym }}^{T_{1} R_{i}}(e)+\mathcal{P}_{\text {sym }}^{T_{2} R_{i}}(e) \text {. }
$$

Utilizing the Taylor series expansion of the exponential function and neglecting the higher order terms, the PDF of $\gamma_{s a_{i}}$ can be approximated as

$$
f_{\gamma_{s a i}}(\gamma) \simeq \frac{1}{\bar{\gamma}_{s a}} .
$$

Thus,

$$
\mathcal{P}_{\text {sym }}^{T_{1} R_{i}}(e) \simeq \frac{\alpha_{z}}{2 \beta_{z}} \frac{1}{\bar{\gamma}_{s a}} \text {. }
$$

Similarly, we obtain the following

$$
\mathcal{P}_{\text {sym }}^{T_{2} R_{i}}(e) \simeq \frac{\alpha_{z}}{2 \beta_{z}} \frac{1}{\bar{\gamma}_{s b}},
$$

and

$$
\mathcal{P}_{\text {sym }}^{T_{1} T_{2}}(e) \simeq \frac{\alpha_{z}}{2 \beta_{z}} \frac{1}{\bar{\gamma}_{h_{1}}} .
$$

The PDF of $\gamma_{h_{1}}$ using the Taylor series expansion can be approximated as

$$
f_{\gamma_{h_{1}}}(\gamma) \simeq \frac{1}{\bar{\gamma}_{h_{1}}}
$$

$f_{\gamma_{b_{t}}}(\gamma)$ can be approximated as

$$
f_{\gamma_{b_{t}}}(\gamma) \simeq k \gamma^{k-1} \frac{1}{\bar{\gamma}_{b}}\left(\frac{1}{\bar{\gamma}_{m}}\right)^{k-1} .
$$

From Eqs. (52) and (53), the PDF of $\gamma_{e_{1}}$ is obtained as

$$
f_{\gamma_{e_{1}}}(\gamma) \simeq \frac{1}{\bar{\gamma}_{h_{1}} \bar{\gamma}_{b}}\left(\frac{1}{\bar{\gamma}_{m}}\right)^{k-1} \gamma^{k}
$$

Thus, substituting Eq. (54) into Eq. (41) and solving the integration using ([40] 3.351.2), we obtain

$$
\mathcal{P}_{\text {sym }}^{T_{1}, \text { coop }}(e \mid k) \simeq \frac{1}{\bar{\gamma}_{h_{1}} \bar{\gamma}_{b}}\left(\frac{1}{\bar{\gamma}_{m}}\right)^{k-1} \frac{\alpha_{x} 2^{k} \Gamma\left(k+\frac{3}{2}\right)}{(k+1) \beta_{x}^{k+1} \sqrt{\pi}},
$$

where $\Gamma(\cdot)$ is the gamma function, given by $\Gamma(u)=$ $\int_{0}^{\infty} e^{-t} t^{u-1} d t$ ([37] 6.1.1). From Eqs. (46), (49), (50), (51), and (55), the asymptotic SEP at the terminal $T_{2}$ for the signal transmitted by $T_{1}$ can be written as

$$
\begin{gathered}
\mathcal{P}_{\text {sym }}^{T_{1}}(e) \simeq\left(\frac{\alpha_{z}}{2 \beta_{z}}\right)^{K+1} \frac{1}{\bar{\gamma}_{h_{1}}}\left(\frac{1}{\bar{\gamma}_{s a}}+\frac{1}{\bar{\gamma}_{s b}}\right)^{K}+\sum_{k=1}^{K}\left(\begin{array}{l}
K \\
k
\end{array}\right) \\
\times \Psi_{k}\left[\frac{\alpha_{z}}{2 \beta_{z}}\left(\frac{1}{\bar{\gamma}_{s a}}+\frac{1}{\bar{\gamma}_{s b}}\right)\right]^{K-k} \frac{1}{\bar{\gamma}_{h_{1}} \bar{\gamma}_{b}}\left(\frac{1}{\bar{\gamma}_{m}}\right)^{k-1},
\end{gathered}
$$

where we define $\Psi_{k}$ as

$$
\Psi_{k}=\frac{\alpha_{x} 2^{k} \Gamma\left(k+\frac{3}{2}\right)}{(k+1) \beta_{x}^{k+1} \sqrt{\pi}} .
$$

Similarly, the asymptotic SEP at the terminal $T_{1}$ for the signal transmitted by $T_{2}, \mathcal{P}_{\text {sym }}^{T_{2}}(e)$, can be obtained by replacing $\bar{\gamma}_{h_{1}}$ with $\bar{\gamma}_{h_{2}}$, and by interchanging $\bar{\gamma}_{b}$ and $\bar{\gamma}_{a}$ in Eq. (56). Thus, the end-to-end asymptotic SEP of the proposed SSC-2W scheme can be written as

$$
\begin{aligned}
& \mathcal{P}_{\text {sym }}^{e 2 e}(e) \simeq \frac{1}{2}\left(\frac{\alpha_{z}}{2 \beta_{z}}\right)^{K+1}\left(\frac{1}{\bar{\gamma}_{h_{1}}}+\frac{1}{\bar{\gamma}_{h_{2}}}\right)\left(\frac{1}{\bar{\gamma}_{s a}}+\frac{1}{\bar{\gamma}_{s b}}\right)^{K} \\
& +\frac{1}{2} \sum_{k=1}^{K}\left(\begin{array}{l}
K \\
k
\end{array}\right) \Psi_{k}\left[\frac{\alpha_{z}}{2 \beta_{z}}\left(\frac{1}{\bar{\gamma}_{s a}}+\frac{1}{\bar{\gamma}_{s b}}\right)\right]^{K-k}\left(\frac{1}{\bar{\gamma}_{h_{1}} \bar{\gamma}_{b}}+\frac{1}{\bar{\gamma}_{h_{2}} \bar{\gamma}_{a}}\right)\left(\frac{1}{\bar{\gamma}_{m}}\right)^{k-1}
\end{aligned}
$$

\subsection{Diversity gain analysis}

To obtain the diversity gain of the proposed SSC-2W scheme, we examine the end-to-end asymptotic SEP, derived in Eq. (58), using equal power allocation (EPA) with $P_{T_{1}}=P_{T_{2}}=P_{R}=P_{e}$, which gives the following

$$
\begin{aligned}
\mathcal{P}_{\text {sym }}^{e 2 e}(e)= & \left(\frac{N_{0}}{P_{e}}\right)^{K+1} \frac{1}{\sigma_{h}^{2}}\left(\frac{1}{\sigma_{a}^{2} \lambda_{T_{1} R}}+\frac{1}{\sigma_{b}^{2} \lambda_{T_{2} R}}\right)^{K} \\
& \times\left[\left(\frac{\alpha_{z}}{2 \beta_{z}}\right)^{K+1}+\sum_{k=1}^{K}\left(\begin{array}{l}
K \\
k
\end{array}\right) \frac{\Psi_{k}}{2}\left(\frac{\alpha_{z}}{2 \beta_{z}}\right)^{K-k}\right] .
\end{aligned}
$$


From Eq. (59), we conclude that the proposed SSC-2W scheme provides a diversity gain of $K+1$, which is one higher than the number of relays in the system.

\section{System optimization}

\subsection{Optimizing relay position under fixed power allocation}

For optimizing the relay position, the normalized distance between the two terminals (i.e., $d_{T_{1} T_{2}}=1$ ) is used throughout this paper. In this section, we consider the scenario that assumes the placement of relays on the straight line, joining both source terminals (i.e., $d_{T_{1} R}=$ $d$ and $d_{T_{2} R}=1-d$ ), in order to reduce the impact of path loss. In addition, we consider the scenario with at least one relay correctly decodes both source signals; otherwise, the optimization problem would be meaningless with $|\mathcal{D}|=0$. Under the condition of fixed power allocated to the source terminals $\left(P_{T_{1}}\right.$ and $\left.P_{T_{2}}\right)$ and the relays $\left(P_{R}\right)$, the relay location optimization problem can be formulated as

$$
\min _{d} \quad \mathcal{P}_{\text {sym }}^{e 2 e}(e)
$$

$$
\text { subject to } \quad 0 \leq d \leq 1 \text {. }
$$

In order to carry out tractable mathematical analysis of all optimization cases, we apply binomial expansion [40] on Eq. (58) and obtain the following form of the endto-end asymptotic SEP, which is used in all optimization analysis,

$$
\begin{aligned}
& \mathcal{P}_{\text {sym }}^{e 2 e}(e) \simeq \frac{1}{2}\left(\frac{\alpha_{z}}{2 \beta_{z}}\right)^{K+1}\left(\frac{1}{\bar{\gamma}_{h_{1}}}+\frac{1}{\bar{\gamma}_{h_{2}}}\right)\left(\frac{1}{\bar{\gamma}_{s a}}+\frac{1}{\bar{\gamma}_{s b}}\right)^{K} \\
& +\frac{\Psi_{K}}{2}\left(\frac{1}{\bar{\gamma}_{h_{1}} \bar{\gamma}_{b}}+\frac{1}{\bar{\gamma}_{h_{2}} \bar{\gamma}_{a}}\right)\left(\frac{1}{\bar{\gamma}_{m}}\right)^{K-1},
\end{aligned}
$$

where

$$
\Psi_{K}=\frac{\alpha_{x} 2^{K} \Gamma\left(K+\frac{3}{2}\right)}{(K+1) \beta_{x}^{K+1} \sqrt{\pi}} .
$$

Hence, Eq. (61) can be written as

$$
\begin{aligned}
\mathcal{P}_{\text {sym }}^{e 2 e}(e)= & \mathcal{A}\left[\mathcal{C} d^{\nu}+\mathcal{H}(1-d)^{\nu}\right]^{K}+\left[\mathcal{J} d^{\nu}+\mathcal{U}(1-d)^{\nu}\right] \\
& \times\left[\mathcal{W} d^{\nu}+\mathcal{Z}(1-d)^{\nu}\right]^{K-1}
\end{aligned}
$$

where we define the following

$$
\begin{aligned}
\mathcal{A} & =\frac{1}{2}\left(\frac{\alpha_{z}}{2 \beta_{z}}\right)^{K+1}\left(\frac{N_{0}}{\sigma_{h}^{2} P_{T_{1}}}+\frac{N_{0}}{\sigma_{h}^{2} P_{T_{2}}}\right), \\
\mathcal{C} & =\frac{N_{0}}{\sigma_{a}^{2} P_{T_{1}}}, \\
\mathcal{H} & =\frac{N_{0}}{\sigma_{b}^{2} P_{T_{2}}}, \\
\mathcal{J} & =\frac{\Psi_{K}}{2} \frac{N_{0}}{\sigma_{h}^{2} P_{T_{2}}} \frac{N_{0}}{\sigma_{a}^{2} P_{R}}, \\
\mathcal{U} & =\frac{\Psi_{K}}{2} \frac{N_{0}}{\sigma_{h}^{2} P_{T_{1}}} \frac{N_{0}}{\sigma_{b}^{2} P_{R}}, \\
\mathcal{W} & =\frac{N_{0}}{\sigma_{a}^{2} P_{R}} \\
\mathcal{Z} & =\frac{N_{0}}{\sigma_{b}^{2} P_{R}} .
\end{aligned}
$$

In order to check the convexity of $\mathcal{P}_{\text {sym }}^{e 2 e}(e)$, given by Eq. (63), we evaluate the second derivative of Eq. (63) with respect to $d$ and it is easily found that $\partial^{2} \mathcal{P}_{\text {sym }}^{e 2 e}(e) / \partial d^{2}$ is positive in the interval $[0,1]$. This shows that the objective function is strictly a convex function of $d$ in the interval $[0,1]$. Thus, taking the first derivative of $\mathcal{P}_{\text {sym }}^{e 2 e}(e)$ with respect to $d$ and equating it to zero, we obtain

$$
\begin{aligned}
& \mathcal{A K}\left[\mathcal{C} d^{v}+\mathcal{H}(1-d)^{\nu}\right]^{K-1}\left[\mathcal{C} v d^{\nu-1}-\mathcal{H} v(1-d)^{\nu-1}\right] \\
& +\left[\mathcal{J} v d^{\nu-1}-\mathcal{U} v(1-d)^{\nu-1}\right]\left[\mathcal{W} d^{v}+\mathcal{Z}(1-d)^{\nu}\right]^{K-1} \\
& +(K-1)\left[\mathcal{J} d^{\nu}+\mathcal{U}(1-d)^{\nu}\right]\left[\mathcal{W} d^{v}+\mathcal{Z}(1-d)^{\nu}\right]^{K-2} \\
& \times\left[\mathcal{W} v d^{\nu-1}-\mathcal{Z} v(1-d)^{\nu-1}\right]=0 .
\end{aligned}
$$

If not impossible, it is very hard to find the closed-form for the optimal relay position $\left(d^{*}\right)$ from Eq. (71); however, simple iterative techniques such as bisection method can be used to find the optimum position of the relay, $d^{*}$.

\subsection{Optimizing power allocation under fixed relay position}

In this section, we investigate the optimal power allocation (OPA) at both source terminals and the relay in order to minimize the average SEP while providing an efficient energy distribution among the participating nodes in the system. For tractable mathematical analysis, we consider the scenario that assumes equal power allocation to the two source terminals (i.e., $P_{T}=P_{T_{1}}=P_{T_{2}}$ ). Under the condition of fixed relay location $\left(d_{T_{1} R}, d_{T_{2} R}\right)$, the power optimization problem can be expressed as 


$$
\begin{aligned}
\min _{P_{T}, P_{R}} & \mathcal{P}_{\mathrm{sym}}^{e 2 e}(e) \\
\text { subject to } & P_{T_{1}}+P_{T_{2}}+P_{R}=2 P_{T}+P_{R} \leq P_{\text {tot }}, \\
& P_{T}, P_{R}>0,
\end{aligned}
$$

where $P_{\text {tot }}$ is the total power budget of the system. We define $\delta$ such that $0<\delta<1$, so that we can express the optimum values of the power allocated to both source terminals and the relay as $P_{T}=\delta P_{\text {tot }} / 2$ and $P_{R}=(1-\delta) P_{\text {tot }}$. Substituting these values into Eq. (61), we obtain

$$
\mathcal{P}_{\text {sym }}^{e 2 e}(e)=\mathcal{I}\left(\frac{1}{\delta}\right)^{K+1}+\mathcal{L}\left(\frac{1}{\delta}\right)\left(\frac{1}{1-\delta}\right)^{K}
$$

where $\mathcal{I}$ and $\mathcal{L}$ are defined as

$$
\begin{aligned}
\mathcal{I}= & \frac{1}{2}\left(\frac{\alpha_{z}}{2 \beta_{z}}\right)^{K+1} \frac{4 N_{0}}{\sigma_{h}^{2} P_{\text {tot }}}\left(\frac{2 N_{0}}{\sigma_{a}^{2} \lambda_{T_{1} R} P_{\text {tot }}}+\frac{2 N_{0}}{\sigma_{b}^{2} \lambda_{T_{2} R} P_{\text {tot }}}\right)^{K}, \\
\mathcal{L}= & \frac{\Psi_{K}}{2} \frac{2 N_{0}}{\sigma_{h}^{2} P_{\text {tot }}}\left(\frac{N_{0}}{\sigma_{a}^{2} \lambda_{T_{1} R} P_{\text {tot }}}+\frac{N_{0}}{\sigma_{b}^{2} \lambda_{T_{2} R} P_{\text {tot }}}\right) \\
& \times\left(\frac{\sigma_{a}^{2} \lambda_{T_{1} R}+\sigma_{b}^{2} \lambda_{T_{2} R}}{\sigma_{a}^{2} \sigma_{b}^{2} \lambda_{T_{1} R} \lambda_{T_{2} R}} \frac{N_{0}}{P_{\text {tot }}}\right)^{K-1} .
\end{aligned}
$$

By taking the second derivative of Eq. (73) with respect to $\delta$, it is easily observed that the objective function is strictly a convex function of $\delta$ in the interval $(0,1)$. Thus, taking the first derivative of Eq. (73) with respect to $\delta$ and equating it to zero, we get

$$
\mathcal{I}(K+1)\left(\frac{1}{\delta}\right)^{K+2}-\mathcal{L} \frac{(K+1) \delta-1}{\delta^{2}}\left(\frac{1}{1-\delta}\right)^{K+1}=0 .
$$

The optimum $\delta^{*}$, obtained using simple iterative techniques such as bisection method, can be used to find the optimum values $P_{T}^{*}$ and $P_{R}^{*}$.

\subsection{Optimizing source power allocation under fixed relay power and position}

Optimum $P_{T}^{*}$, obtained using OPA, can be used to evaluate the optimal source power allocation (OSA) (i.e., $\left.P_{T_{1}}^{*}, P_{T_{2}}^{*}\right)$ under the condition of fixed relay power $\left(P_{R}\right)$ and position $\left(d_{T_{1} R}, d_{T_{2} R}\right)$. The source power optimization problem can be mathematically stated as

$$
\begin{aligned}
\min _{P_{T_{1}}, P_{T_{1}}} & \mathcal{P}_{\text {sym }}^{e 2 e}(e) \\
\text { subject to } & P_{T_{1}}+P_{T_{2}} \leq P_{T S}, \\
& P_{T_{1}}, P_{T_{2}}>0,
\end{aligned}
$$

where $P_{T S}$ is the total power allocated to the source terminals of the system. By defining $\rho$ such that $0<\rho<1$, we can represent the optimum values of the power allocated to the terminals as $P_{T_{1}}=\rho P_{T S}$ and $P_{T_{2}}=(1-\rho) P_{T S}$. Substituting these values into (61), we get

$\mathcal{P}_{\text {sym }}^{e 2 e}(e)=\mathcal{B}\left(\frac{1}{\rho}+\frac{1}{1-\rho}\right)\left(\frac{\mathcal{V}}{\rho}+\frac{\mathcal{X}}{1-\rho}\right)^{K}+\mathcal{Y}\left(\frac{\mathcal{V}}{\rho}+\frac{\mathcal{X}}{1-\rho}\right)$,

where we define the following

$$
\begin{aligned}
& \mathcal{B}=\frac{1}{2}\left(\frac{\alpha_{z}}{2 \beta_{z}}\right)^{K+1} \frac{N_{0}}{\sigma_{h}^{2} P_{T S}}, \\
& \mathcal{V}=\frac{N_{0}}{\sigma_{a}^{2} \lambda_{T_{1} R} P_{T S}}, \\
& \mathcal{X}=\frac{N_{0}}{\sigma_{b}^{2} \lambda_{T_{2} R} P_{T S}}, \\
& \mathcal{Y}=\frac{\Psi_{K}}{2} \frac{N_{0}}{\sigma_{h}^{2} P_{R}}\left(\frac{\sigma_{a}^{2} \lambda_{T_{1} R}+\sigma_{b}^{2} \lambda_{T_{2} R}}{\sigma_{a}^{2} \sigma_{b}^{2} \lambda_{T_{1} R} \lambda_{T_{2} R}} \frac{N_{0}}{P_{R}}\right)^{K-1} .
\end{aligned}
$$

The expression for $\mathcal{P}_{\text {sym }}^{e 2 e}(e)$, given by Eq. (78), is found to be strictly a convex function of $\rho$ in the interval $(0,1)$. Thus, taking the first derivative of Eq. (78) with respect to $\rho$ and equating it to zero, we obtain

$\mathcal{B} \frac{(2 \rho-1)}{\rho^{2}(1-\rho)^{2}}\left(\frac{\mathcal{V}}{\rho}+\frac{\mathcal{X}}{1-\rho}\right)^{K}+\mathcal{Y}\left[\frac{\mathcal{X}}{(1-\rho)^{2}}-\frac{\mathcal{V}}{\rho^{2}}\right]$
$+\mathcal{B} K \frac{1}{\rho(1-\rho)}\left[\frac{\mathcal{X}}{(1-\rho)^{2}}-\frac{\mathcal{V}}{\rho^{2}}\right]\left(\frac{\mathcal{V}}{\rho}+\frac{\mathcal{X}}{1-\rho}\right)^{K-1}=0$.

The optimum value of $\rho\left(\rho^{*}\right)$ can be obtained from the previous expression using simple iterative techniques such as bisection method, and hence, the optimum values of the power allocated to the source terminals (i.e., $P_{T_{1}}^{*}, P_{T_{2}}^{*}$ ) can be calculated.

\section{Outage probability analysis}

In this section, we evaluate the overall outage performance of the proposed SSC-2W scheme over Rayleigh fading channel. We also derive a closed-form expression for the asymptotic approximation of the outage probability.

\subsection{Exact outage probability}

For the transmission scenario presented in Section 2, a total system outage occurs either when $T_{1} \rightarrow T_{2}, T_{2} \rightarrow T_{1}$, $\mathrm{T}_{1} \rightarrow \mathrm{R}_{i}$, and $\mathrm{T}_{2} \rightarrow \mathrm{R}_{i}$ links are in outage or when the cooperative links are in outage assuming no outage in $\mathrm{T}_{1} \rightarrow \mathrm{R}_{i}$ and $\mathrm{T}_{2} \rightarrow \mathrm{R}_{i}$ links. Thus, the outage probability for the signal transmitted by $T_{1}$ can be expressed as

$$
\mathcal{P}_{\text {out }}^{T_{1}}=\left(\mathcal{P}_{\text {out }}^{\text {off }}\right)^{K} \mathcal{P}_{\text {out }}^{T_{1} T_{2}}+\left(1-\mathcal{P}_{\text {out }}^{\text {off }}\right)^{K} \mathcal{P}_{\text {out }}^{T_{1} \text {,coop }},
$$

where $\mathcal{P}_{\text {out }}^{T_{1} T_{2}}$ denotes the outage probability of the $T_{1} \rightarrow$ $\mathrm{T}_{2}$ link, $\mathcal{P}_{\text {out }}^{T_{1} \text {,coop }}$ denotes the outage probability of the 
cooperative link, and $\mathcal{P}_{\text {out }}^{\text {off }}$ denotes the outage probability when a relay link to both terminals has failed. $\mathcal{P}_{\text {out }}^{\text {off }}$ can be written as

$$
\mathcal{P}_{\text {out }}^{\text {off }}=1-\left(1-\mathcal{P}_{\text {out }}^{T_{1} R}\right)\left(1-\mathcal{P}_{\text {out }}^{T_{2} R}\right)
$$

where $\mathcal{P}_{\text {out }}^{T_{1} R}$ and $\mathcal{P}_{\text {out }}^{T_{2} R}$ represent the outage probabilities of $\mathrm{T}_{1} \rightarrow \mathrm{R}_{i}$ and $\mathrm{T}_{2} \rightarrow \mathrm{R}_{i}$ links, respectively. For a given target rate $R$ bits $/ \mathrm{s} / \mathrm{Hz}$, the event that an outage happens in the $\mathrm{T}_{1} \rightarrow \mathrm{R}_{i}$ link corresponds to the event $(1 / 3) \log _{2}(1+$ $\left.\gamma_{s a_{i}}\right)<R$, or equivalently $\gamma_{s a_{i}}<\mu_{t h}$, where $\mu_{t h}=2^{3 R}-1$. The reason for the $1 / 3$ factor is that there are 3 time slots for transmission. Thus, $\mathcal{P}_{\text {out }}^{T_{1} R}$ can be expressed as

$$
\begin{aligned}
& \mathcal{P}_{\text {out }}^{T_{1} R} \triangleq \operatorname{Pr}\left\{\frac{1}{3} \log _{2}\left(1+\gamma_{s a_{i}}\right)<R\right\}, \\
& \mathcal{P}_{\text {out }}^{T_{1} R}=1-\exp \left(-\frac{\mu_{t h}}{\bar{\gamma}_{\text {sa }}}\right) .
\end{aligned}
$$

Similarly, $\mathcal{P}_{\text {out }}^{T_{2} R}$ and $\mathcal{P}_{\text {out }}^{T_{1} T_{2}}$ can be obtained as

$$
\mathcal{P}_{\text {out }}^{T_{2} R}=1-\exp \left(-\frac{\mu_{t h}}{\bar{\gamma}_{s b}}\right)
$$

and

$$
\mathcal{P}_{\text {out }}^{T_{1} T_{2}}=1-\exp \left(-\frac{\mu_{t h}}{\bar{\gamma}_{h_{1}}}\right) .
$$

When no outage happens in $\mathrm{T}_{1} \rightarrow \mathrm{R}_{i}$ and $\mathrm{T}_{2} \rightarrow \mathrm{R}_{i}$ links and the relay successfully decodes both source signals, the best relay transmits different symbols through $\mathrm{R}_{t} \rightarrow \mathrm{T}_{1}$ and $\mathrm{R}_{t} \rightarrow \mathrm{T}_{2}$ links to both source terminals in the third time slot. Thus, the average mutual information for the cooperative link at the terminal $T_{2}$, for the signal transmitted by $T_{1}$, is given by

$$
I_{T_{1}}^{\mathrm{coop}} \triangleq \frac{1}{3} \log _{2}\left(1+\gamma_{h_{1}}\right)+\frac{1}{3} \log _{2}\left(1+\gamma_{b_{t}}\right) \text {. }
$$

Assuming $\gamma_{h_{1}}$ and $\gamma_{b_{t}}$ as independent random variables, the outage probability of the cooperative link at the terminal $T_{2}$, for the signal transmitted by $T_{1}$, can be expressed as

$$
\mathcal{P}_{\text {out }}^{T_{1}, \text { coop }}=\operatorname{Pr}\left\{I_{T_{1}}^{\text {coop }}<R\right\}=\iint_{\Lambda} f\left(\gamma_{h_{1}}, \gamma_{b_{t}}\right) d \gamma_{h_{1}} d \gamma_{b_{t}},
$$

where $\Lambda \triangleq\left\{\left(\gamma_{h_{1}}, \gamma_{b_{t}}\right) \mid\left(1+\gamma_{h_{1}}\right)\left(1+\gamma_{b_{t}}\right)<2^{3 R}, \quad \gamma_{h_{1}} \geq\right.$ $\left.0, \gamma_{b_{t}} \geq 0\right\}$ and $f\left(\gamma_{h_{1}}, \gamma_{b_{t}}\right)=f_{\gamma_{h_{1}}}\left(\gamma_{h_{1}}\right) f_{\gamma_{b_{t}}}\left(\gamma_{b_{t}}\right)$ is the joint PDF of $\gamma_{h_{1}}$ and $\gamma_{b_{t}}$. Thus,

$$
\mathcal{P}_{\text {out }}^{T_{1} \text {,coop }}=\int_{0}^{\mu_{\text {th }}} \int_{0}^{\frac{1+\mu_{t h}}{1+\gamma_{h_{1}}}-1} f_{\gamma_{h_{1}}}\left(\gamma_{h_{1}}\right) f_{\gamma_{b_{t}}}\left(\gamma_{b_{t}}\right) d \gamma_{h_{1}} d \gamma_{b_{t}} .
$$

Using the Taylor series to approximate terms such as $\exp \left\{-\left(\mu_{t h}-\gamma_{h_{1}}\right) /\left(\bar{\gamma}_{b}\left(1+\gamma_{h_{1}}\right)\right)\right\}$ and solving the integral in the previous expression, we obtain $\mathcal{P}_{\text {out }}^{T_{1} \text {,coop }}$ as

$$
\begin{aligned}
& \mathcal{P}_{\text {out }}^{T_{1} \text {,coop }}=\sum_{k=1}^{K}\left(\begin{array}{l}
K \\
k
\end{array}\right) \frac{(-1)^{k-1}}{\bar{\gamma}_{a}} \frac{\bar{\gamma}_{m}}{k \bar{\gamma}_{b}-\bar{\gamma}_{m}}\left[1-\exp \left(-\frac{\mu_{t h}}{\bar{\gamma}_{h_{1}}}\right)\right] \\
& {\left[k \bar{\gamma}_{b}\left\{1-\exp \left(-\frac{\mu_{t h}}{\bar{\gamma}_{b}}\right)\right\}-\bar{\gamma}_{m}\left\{1-\exp \left(-\frac{k \mu_{t h}}{\bar{\gamma}_{m}}\right)\right\}\right]} \\
& +\sum_{k=1}^{K}\left(\begin{array}{l}
K \\
k
\end{array}\right) \frac{(-1)^{k-1}}{\bar{\gamma}_{b}} \bar{\gamma}_{m}\left[1-\exp \left(-\frac{\mu_{t h}}{\bar{\gamma}_{h_{1}}}\right)\right]\left[1-\exp \left(-\frac{k \mu_{t h}}{\bar{\gamma}_{m}}\right)\right] .
\end{aligned}
$$

From Eqs. (84), (85), (87), (88), (89), and (93), we can obtain the outage probability for the signal transmitted by $T_{1}, \mathcal{P}_{\text {out }}^{T_{1}}$. Similarly, the outage probability for the signal transmitted by $\mathrm{T}_{2}, \mathcal{P}_{\text {out }}^{T_{2}}$, can be obtained by replacing $\bar{\gamma}_{h_{1}}$ with $\bar{\gamma}_{h_{2}}$ and by interchanging $\bar{\gamma}_{b}$ and $\bar{\gamma}_{a}$ in $\mathcal{P}_{\text {out }}^{T_{1}}$. Thus, the overall outage probability of the system is given by

$$
\mathcal{P}_{\text {out }}=\frac{\mathcal{P}_{\text {out }}^{T_{1}}+\mathcal{P}_{\text {out }}^{T_{2}}}{2}
$$

\subsection{Asymptotic outage probability}

In the following, we evaluate the asymptotic approximation of the overall outage probability, which is valid for high SNR and provides insight into the system performance. Using the fact that $\left(1-\mathcal{P}_{\text {out }}^{\text {ouf }}\right) \rightarrow 1$ for high SNR, the asymptotic outage probability of $T_{1}$ can be expressed as

$$
\mathcal{P}_{\text {out }}^{T_{1}} \simeq\left(\mathcal{P}_{\text {out }}^{\text {off }}\right)^{K} \mathcal{P}_{\text {out }}^{T_{1} T_{2}}+\mathcal{P}_{\text {out }}^{T_{1} \text {,coop }},
$$

where $\mathcal{P}_{\text {out }}^{\text {off }}$ can be approximated as

$$
\mathcal{P}_{\text {out }}^{\text {off }} \approx \mathcal{P}_{\text {out }}^{T_{1} R}+\mathcal{P}_{\text {out }}^{T_{2} R}
$$

Using Eq. (48), $\mathcal{P}_{\text {out }}^{T_{1} R}$ can be approximated as

$$
\mathcal{P}_{\text {out }}^{T_{1} R} \simeq \frac{\mu_{t h}}{\bar{\gamma}_{s a}}
$$

Similarly,

$$
\mathcal{P}_{\text {out }}^{T_{2} R} \simeq \frac{\mu_{t h}}{\bar{\gamma}_{s b}}
$$

and

$$
\mathcal{P}_{\text {out }}^{T_{1} T_{2}} \simeq \frac{\mu_{t h}}{\bar{\gamma}_{h_{1}}}
$$

Substituting Eqs. (52) and (53) into Eq. (92), and solving the integration, $\mathcal{P}_{\text {out }}^{T_{1} \text {,coop }}$ is approximated as

$$
\mathcal{P}_{\text {out }}^{T_{1} \text {,coop }} \simeq \frac{1}{\bar{\gamma}_{h_{1}} \bar{\gamma}_{b}}\left(\frac{1}{\bar{\gamma}_{m}}\right)^{K-1} \frac{\mu_{t h}^{K+1}}{K+1} .
$$


Thus, combining Eqs, (95)-(100), the asymptotic outage probability of $\mathrm{T}_{1}$ can be written as

$$
\mathcal{P}_{\text {out }}^{T_{1}} \simeq\left(\frac{\mu_{t h}}{\bar{\gamma}_{s a}}+\frac{\mu_{t h}}{\bar{\gamma}_{s b}}\right)^{K} \frac{\mu_{t h}}{\bar{\gamma}_{h_{1}}}+\frac{1}{\bar{\gamma}_{h_{1}} \bar{\gamma}_{b}}\left(\frac{1}{\bar{\gamma}_{m}}\right)^{K-1} \frac{\mu_{t h}^{K+1}}{K+1} .
$$

In a similar manner, the asymptotic outage probability of $\mathrm{T}_{2}, \mathcal{P}_{\text {out }}^{T_{2}}$, can be obtained by replacing $\bar{\gamma}_{h_{1}}$ with $\bar{\gamma}_{h_{2}}$, and by interchanging $\bar{\gamma}_{b}$ and $\bar{\gamma}_{a}$ in Eq. (101). The overall asymptotic outage probability of the system can be written as

$$
\begin{aligned}
\mathcal{P}_{\text {out }} & \simeq\left(\frac{\mu_{t h}}{\bar{\gamma}_{s a}}+\frac{\mu_{t h}}{\bar{\gamma}_{s b}}\right)^{K}\left(\frac{\mu_{t h}}{\bar{\gamma}_{h_{1}}}+\frac{\mu_{t h}}{\bar{\gamma}_{h_{2}}}\right) \\
& +\left(\frac{1}{\bar{\gamma}_{h_{1}} \bar{\gamma}_{b}}+\frac{1}{\bar{\gamma}_{h_{2}} \bar{\gamma}_{a}}\right)\left(\frac{1}{\bar{\gamma}_{m}}\right)^{K-1} \frac{\mu_{t h}^{K+1}}{K+1} .
\end{aligned}
$$

\section{Channel capacity analysis}

\subsection{Average channel capacity}

In this section, the average channel capacity, in the Shannon's sense, is evaluated by averaging the instantaneous capacity for an AWGN channel over the fading distribution for optimal rate adaptation with constant transmit power and receiver channel state information $[39,42]$. Thus, the average channel capacity of the proposed SSC-2W scheme can be expressed as

$$
\bar{C}=\bar{C}_{T_{1} T_{2}}+\bar{C}_{T_{2} T_{1}}+\bar{C}_{R_{t} T_{1}}+\bar{C}_{R_{t} T_{2}},
$$

where $\bar{C}_{T_{1} T_{2}}, \bar{C}_{T_{2} T_{1}}, \bar{C}_{R_{t} T_{1}}$, and $\bar{C}_{R_{t} T_{2}}$ denote the average channel capacities of $\mathrm{T}_{1} \rightarrow \mathrm{T}_{2}, \mathrm{~T}_{2} \rightarrow \mathrm{T}_{1}, \mathrm{R}_{t} \rightarrow \mathrm{T}_{1}$, and $R_{t} \rightarrow T_{2}$ links, respectively. The average channel capacity of the $T_{1} \rightarrow T_{2}$ link can be written as

$$
\bar{C}_{T_{1} T_{2}}=B \frac{1}{3} \int_{0}^{\infty} \log _{2}\left(1+\gamma_{h_{1}}\right) f_{\gamma_{h_{1}}}\left(\gamma_{h_{1}}\right) d \gamma_{h_{1}},
$$

where $B$ is the bandwidth of the channel. The reason for the $1 / 3$ factor is that one symbol is transmitted through the $T_{1} \rightarrow T_{2}$ link in three time slots. In order to simplify Eq. (104), we formulate the following relation using ([40] 3.352.4)

$$
\int_{0}^{\infty} \ln (1+x) e^{-x / c} d x=c e^{1 / c} E_{1}\left(\frac{1}{c}\right),
$$

where $E_{1}(\cdot)$ represents the exponential integral, defined as $E_{1}(u)=\int_{u}^{\infty}(\exp (-t) / t) d t$ ([37] 5.1.1). Substituting $f_{\gamma_{h_{1}}}\left(\gamma_{h_{1}}\right)$ into Eq. (104), and solving the integration using Eq. (105), $\bar{C}_{T_{1} T_{2}}$ can be written as

$$
\bar{C}_{T_{1} T_{2}}=B \frac{1}{3 \ln 2} e^{1 / \bar{\gamma}_{h_{1}} E_{1}}\left(\frac{1}{\bar{\gamma}_{h_{1}}}\right) .
$$

Similarly, we can obtain

$$
\bar{C}_{T_{2} T_{1}}=B \frac{1}{3 \ln 2} e^{1 / \bar{\gamma}_{h_{2}} E_{1}}\left(\frac{1}{\bar{\gamma}_{h_{2}}}\right) .
$$

The average channel capacity of the $R_{t} \rightarrow T_{2}$ link can be written as

$$
\bar{C}_{R_{t} T_{2}}=B \frac{1}{3} \int_{0}^{\infty} \log _{2}\left(1+\gamma_{b_{t}}\right) f_{\gamma_{b_{t}}}\left(\gamma_{b_{t}}\right) d \gamma_{b_{t}} .
$$

Substituting Eq. (42) into Eq. (108), and solving the integration using Eq. (105), $\bar{C}_{R_{t} T_{2}}$ can be expressed as

$$
\begin{aligned}
& \bar{C}_{R_{t} T_{2}}=\frac{B}{3 \ln 2} \sum_{k=1}^{K}\left(\begin{array}{c}
K \\
k
\end{array}\right) \frac{(-1)^{k-1}}{\bar{\gamma}_{a}} \frac{k \bar{\gamma}_{m}}{k \bar{\gamma}_{b}-\bar{\gamma}_{m}} \times \\
& {\left[\bar{\gamma}_{b} e^{1 / \bar{\gamma}_{b}} E_{1}\left(\frac{1}{\bar{\gamma}_{b}}\right)-\frac{\bar{\gamma}_{m}}{k} e^{k / \bar{\gamma}_{m}} E_{1}\left(\frac{k}{\bar{\gamma}_{m}}\right)\right]} \\
& +\frac{B}{3 \ln 2} \sum_{k=1}^{K}\left(\begin{array}{l}
K \\
k
\end{array}\right) \frac{(-1)^{k-1}}{\bar{\gamma}_{b}} \bar{\gamma}_{m} e^{k / \bar{\gamma}_{m}} E_{1}\left(\frac{k}{\bar{\gamma}_{m}}\right) .
\end{aligned}
$$

Similarly, the average channel capacity of the $\mathrm{R}_{t} \rightarrow \mathrm{T}_{1}$ link, $\bar{C}_{R_{t} T_{1}}$, can be obtained by interchanging $\bar{\gamma}_{b}$ and $\bar{\gamma}_{a}$ in Eq. (109). Thus, the closed-form expression for the average channel capacity of the system can be expressed as

$$
\begin{aligned}
\frac{\bar{C}}{B}= & \frac{1}{3 \ln 2} \sum_{k=1}^{K}\left(\begin{array}{l}
K \\
k
\end{array}\right) \frac{(-1)^{k-1}}{\bar{\gamma}_{a}} \frac{k \bar{\gamma}_{m}}{k \bar{\gamma}_{b}-\bar{\gamma}_{m}} \\
& \times\left[\bar{\gamma}_{b} e^{1 / \bar{\gamma}_{b}} E_{1}\left(\frac{1}{\bar{\gamma}_{b}}\right)-\frac{\bar{\gamma}_{m}}{k} e^{k / \bar{\gamma}_{m}} E_{1}\left(\frac{k}{\bar{\gamma}_{m}}\right)\right] \\
& +\frac{1}{3 \ln 2} \sum_{k=1}^{K}\left(\begin{array}{l}
K \\
k
\end{array}\right) \frac{(-1)^{k-1}}{\bar{\gamma}_{b}} \frac{k \bar{\gamma}_{m}}{k \bar{\gamma}_{a}-\bar{\gamma}_{m}} \\
& \times\left[\bar{\gamma}_{a} e^{1 / \bar{\gamma}_{a}} E_{1}\left(\frac{1}{\bar{\gamma}_{a}}\right)-\frac{\bar{\gamma}_{m}}{k} e^{k / \bar{\gamma}_{m}} E_{1}\left(\frac{k}{\bar{\gamma}_{m}}\right)\right] \\
& +\frac{1}{3 \ln 2} \sum_{k=1}^{K}\left(\begin{array}{l}
K \\
k
\end{array}\right)(-1)^{k-1} e^{k / \bar{\gamma}_{m}} E_{1}\left(\frac{k}{\bar{\gamma}_{m}}\right) \\
+ & \frac{1}{3 \ln 2}\left[e^{1 / \bar{\gamma}_{h_{1}}} E_{1}\left(\frac{1}{\bar{\gamma}_{h_{1}}}\right)+e^{\left.1 / \bar{\gamma}_{h_{2}} E_{1}\left(\frac{1}{\bar{\gamma}_{h_{2}}}\right)\right] .}\right.
\end{aligned}
$$

\subsection{Upper bound on the capacity}

Using Jensen's inequality, the average channel capacity of a link can be upper bounded as

$$
\bar{C} \leq B \frac{1}{3} \log _{2}(1+\mathbb{E}\{\gamma\}),
$$

where $\mathbb{E}\{\cdot\}$ denotes the expectation operator and is evaluated by $\mathbb{E}\{\gamma\}=\int_{0}^{\infty} \gamma f_{\gamma}(\gamma) d \gamma$ using $\int_{0}^{\infty} t e^{-t / c} d t=c^{2}$. Thus, we obtain the upper bounds on the capacities of different links as

$$
\begin{aligned}
& \bar{C}_{T_{1} T_{2}} \leq B \frac{1}{3} \log _{2}\left(1+\bar{\gamma}_{h_{1}}\right), \\
& \bar{C}_{T_{2} T_{1}} \leq B \frac{1}{3} \log _{2}\left(1+\bar{\gamma}_{h_{2}}\right),
\end{aligned}
$$


and

$$
\bar{C}_{R_{t} T_{2}} \leq B \frac{1}{3} \log _{2}\left(1+\mathbb{E}\left\{\gamma_{b_{t}}\right\}\right),
$$

where

$$
\begin{aligned}
\mathbb{E}\left\{\gamma_{b_{t}}\right\}= & \sum_{k=1}^{K}\left(\begin{array}{l}
K \\
k
\end{array}\right) \frac{(-1)^{k-1}}{\bar{\gamma}_{a}} \frac{k \bar{\gamma}_{m}}{k \bar{\gamma}_{b}-\bar{\gamma}_{m}} \\
& \times\left[\bar{\gamma}_{b}^{2}-\left(\frac{\bar{\gamma}_{m}}{k}\right)^{2}\right]+\sum_{k=1}^{K}\left(\begin{array}{l}
K \\
k
\end{array}\right) \frac{(-1)^{k-1}}{\bar{\gamma}_{b}} \frac{\bar{\gamma}_{m}^{2}}{k} .
\end{aligned}
$$

Similarly, $\bar{C}_{R_{t} T_{1}}$ can be obtained by interchanging $\bar{\gamma}_{b}$ and $\bar{\gamma}_{a}$ in Eq. (115). Then, the upper bound on the capacity of the system can be easily obtained.

\section{Performance results}

In this section, we present numerical results to illustrate the performance of the proposed SSC-2W scheme using derived analytical expressions and numerical simulations. These include bit error probability (BEP), outage probability, and channel capacity of the proposed SSC-2W scheme over a slow Rayleigh fading channel as a function of system parameters such as the number of relays $(K)$, average signal-to-noise ratio $\left(E_{b} / N_{0}\right)$, information rate $(R)$, equal power allocation (EPA), optimal power allocation (OPA), and optimal relay position (ORP). The analytical results are validated using Monte Carlo simulations, which were performed with $10^{9}$ trials for each simulation point for accuracy and correctness. The SSD scheme is using 4-QAM modulation with rotation angle of $26.6^{\circ}$ [31]. The path loss exponent is set as $v=3$ and the variances are $\sigma_{h}^{2}=\sigma_{a}^{2}=\sigma_{b}^{2}=1$. The studied system is assumed to have $K$ relays located at the middle of the two source terminals, unless otherwise stated. For the fair analysis, the total power budget to exchange four symbols in the proposed SSC-2W scheme is equivalent to that of the direct transmission, thus $P_{T_{1}}=P_{T_{2}}=1$ for first and second time slots and $P_{R}=2$ for the third time slot, making the total power budget equals to four units.

Figure 3 shows the BEP of the system as a function of the average SNR $\left(E_{b} / N_{0}\right)$ for different numbers of relays $(K)$. The simulation results in this plot are represented by $\diamond$ markers while analytical and asymptotic results are shown by solid and dashed lines, respectively. In addition, the error performance of the proposed SSC-2W scheme is compared with the conventional three-phase two-way DF relaying system with one relay $(K=1)$, which is shown by the $\circ$ markers. It is evident from the figure that the proposed SSC-2W scheme with one relay has almost the same performance as the conventional relaying system with one relay. It is clear from this figure that analytical results for the error probability, derived from Eq. (45), are in perfect agreement with the simulation results, thus validating our

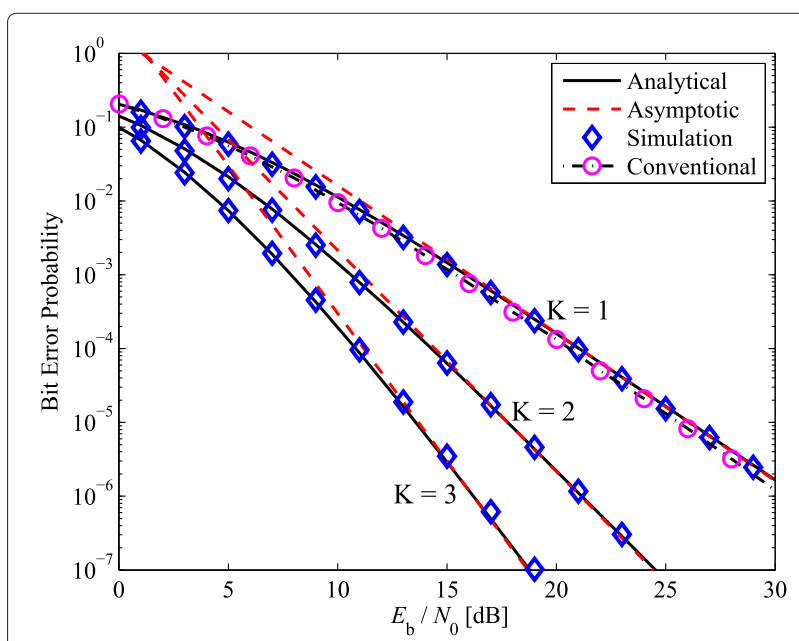

Fig. 3 Bit error probability as a function of $E_{b} / N_{0}$

mathematical derivations. Also, the asymptotic results, derived in Eq. (58), are tight and have a good match with the analytical and simulation results at medium and high SNRs. It is observed that as the number of relays $(K)$ increases, the slope of the BEP curve becomes steeper, indicating the increase in cooperative diversity gain. This indicates that the diversity gain achieved is $K+1$, which is given by Eq. (59). For example, $\mathrm{BEP}=10^{-5}$ can be achieved at $E_{b} / N_{0}=26 \mathrm{~dB}$ with one relay $(K=1)$, or at $E_{b} / N_{0}=17.7 \mathrm{~dB}$ with two relays $(K=2)$, or at $E_{b} / N_{0}=13.5 \mathrm{~dB}$ with three relays $(K=3)$. Thus, compared to the single-relay system, an $E_{b} / N_{0}$ gain of about 8.3 and $12.5 \mathrm{~dB}$ is achieved with two and three relays, respectively.

Figure 4 compares the error performance of the system for different optimization schemes when relays are placed

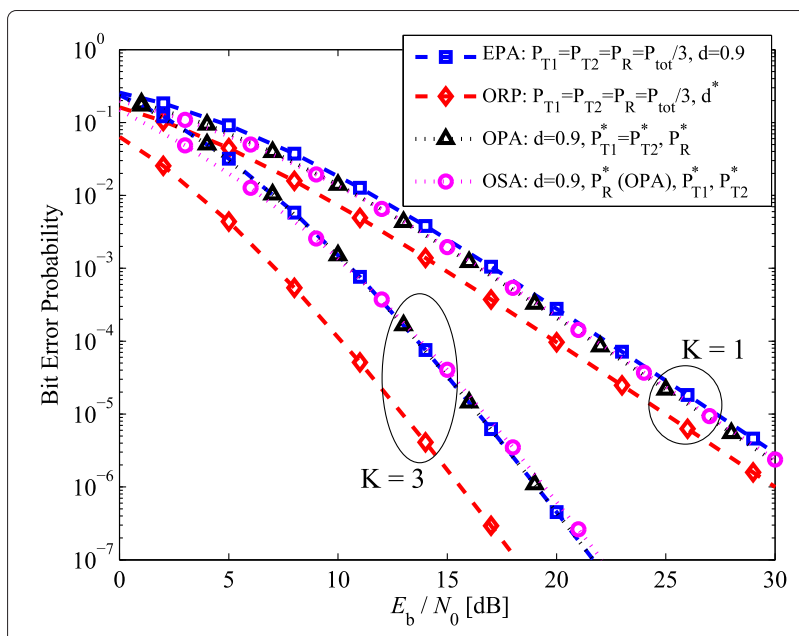

Fig. 4 Bit error probability as a function of $E_{b} / N_{0}$, with different optimization schemes at $d_{T_{1} R}=0.9$ 
close to the terminal $T_{2}$ and far away from the terminal $\mathrm{T}_{1}$ (i.e., $d_{T_{1} R}=0.9$ ). The figure also shows the average BEP of the system for EPA, ORP, OPA, and OSA. A clear performance enhancement with optimal relay position is observed over other optimization schemes and about $4 \mathrm{~dB}$ gain in $E_{b} / N_{0}$ is achieved. The optimum placement of relays is obtained using Eq. (71), which is at the middle location $\left(d^{*}=0.5\right)$ due to the two-way communication of the two source terminals. It is observed that when relays are close to either source terminal, the optimal power scheme allocates more power to the terminal farther from the relays, which supports to maintain the error performance.

The outage performance of the system as a function of $E_{b} / N_{0}$ is shown in Fig. 5. It is evident that the simulation results perfectly match with the analytical results for the outage probability, derived from Eq. (94), which confirms the accuracy of our mathematical derivations for the outage probability. Also, it is noted that the asymptotic results for the outage, derived from Eq. (102), have good agreement with both simulation and analytical results at medium and high SNRs. The slope of the outage probability becomes steeper with the increase in the number of relays, which implies an increase in the diversity order of the system.

Figure 6 depicts the outage probability as a function of the information rate $(R)$ at $E_{b} / N_{0}=20 \mathrm{~dB}$. Again, it is noted that both exact and asymptotic outage probability results, Eqs. (94) and (102), are in close agreement with the simulation results.

Figure 7 demonstrates the average channel capacity of the system as a function of $E_{b} / N_{0}$. The figure also shows the upper bound on the capacity with dashed lines. Channel capacity results for $K=1$ and $K=3$ are shown in the figure and channel capacity for direct transmission is also plotted for comparison. It is evident that the analytical

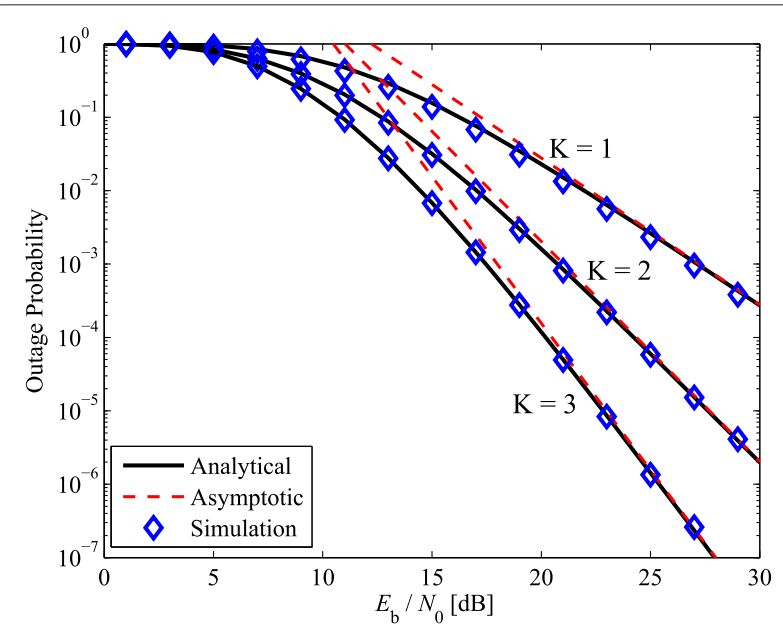

Fig. 5 Outage probability as a function of $E_{b} / N_{0}$ when $R=2$

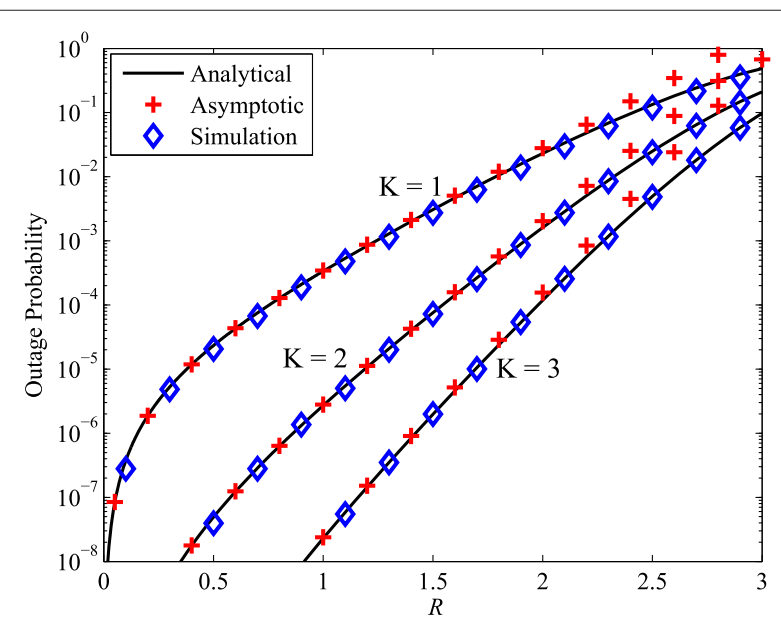

Fig. 6 Outage probability as a function of information rate $(R)$ at $E_{b} / N_{0}=20 \mathrm{~dB}$

expressions of the capacity, derived from Eq. (110) as well as in Section 6.2, are in excellent agreement with their corresponding simulation results, thus validating our analytical work for channel capacity. The figure shows the significant capacity improvement with the proposed SSC$2 \mathrm{~W}$ scheme, as the proposed scheme exchanges four symbols in three time slots, while direct transmission requires four time slots to exchange the same four symbols. Moreover, the capacity also improves with the increase in the number of relays.

\section{Conclusions}

In this paper, an SSD-based two-way DF relaying system using the best relay is presented and its performance was analyzed. The error probability expression of the proposed SSC-2W scheme was derived over the Rayleigh

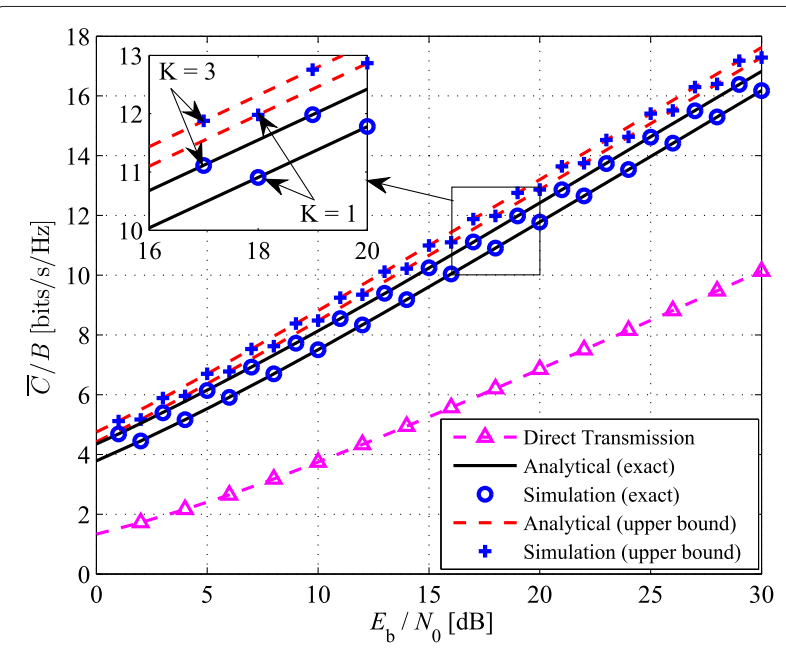

Fig. 7 Channel capacity as a function of $E_{b} / N_{0}$ 
fading channel. It was shown that the proposed SSC-2W scheme enhances the performance and doubles the spectral efficiency of the conventional three-phase two-way DF relaying system. The closed-form expression for the asymptotic error probability was obtained, which showed that the proposed SSC-2W scheme can achieve a diversity order equal to one higher than the number of cooperating relays. A detailed analysis of system optimization, including relay placement and power allocation, was additionally conducted. It was shown that the power allocation is affected by the relay position, especially when the relays are close to either source terminal. Moreover, the system optimization was shown to enhance the performance significantly. Exact and asymptotic outage probability expressions were also derived. Lastly, closed-form expressions for average channel capacity as well as an upper bound on channel capacity were obtained. The validity of all of the aforementioned analysis and derivations was confirmed through extensive Monte Carlo simulations.

\section{Competing interests}

The authors declare that they have no competing interests.

Received: 24 December 2015 Accepted: 19 May 2016

Published online: 31 May 2016

\section{References}

1. MK Müller, M Taranetz, M Rupp, Providing current and future cellular services to high speed trains. IEEE Commun. Mag. 53(10), 96-101 (2015)

2. D Hwang, S-G Hong, T-J Lee, Multiuser two way relaying schemes in the future cellular network. IEEE Trans. Wireless Commun. 12(10), 5200-5207 (2013)

3. $\mathrm{XTaO}, \mathrm{XXu}, \mathrm{Q}$ Cui, An overview of cooperative communications. IEEE Commun. Mag. 50(6), 65-71 (2012)

4. T-T Tran, Y Shin, O-S Shin, Overview of enabling technologies for 3GPP LTE-advanced. EURASIP J. Wirel. Commun. Netw. 2012(1), 1-12 (2012)

5. A Sendonaris, E Erkip, B Aazhang, User cooperation diversity. Part I. System description. IEEE Trans. Commun. 51(11), 1927-1938 (2003)

6. W Zhuang, M Ismail, Cooperation in wireless communication networks. IEEE Wireless Commun. 19(2), 10-20 (2012)

7. JN Laneman, DNC Tse, GW Wornell, Cooperative diversity in wireless networks: efficient protocols and outage behavior. IEEE Trans. Inf. Theory. 50(12), 3062-3080 (2004)

8. TE Hunter, A Nosratinia, Diversity through coded cooperation. IEEE Trans. Wireless Commun. 5(2), 283-289 (2006)

9. G Kramer, M Gastpar, P Gupta, Cooperative strategies and capacity theorems for relay networks. IEEE Trans. Inf. Theory. 51(9), 3037-3063 (2005)

10. H Chen, AB Gershman, S ShahbazPanahi, Filter-and-forward distributed beamforming in relay networks with frequency selective fading. IEEE Trans. Signal Process. 58(3), 1251-1262 (2010)

11. KJ Rayliu, AK Sadek, W Su, A Kwasinksi, Cooperative Communications and Networking. (Cambrdige University Press, Cambridge, UK, 2009)

12. A Nosratinia, TE Hunter, A Hedayat, Cooperative communication in wireless networks. IEEE Commun. Mag. 42(10), 74-80 (2004)

13. M Cardone, D Tuninetti, R Knopp, U Salim, On the gaussian half-duplex relay channel. IEEE Trans. Inf. Theory. 60(5), 2542-2562 (2014)

14. B Rankov, A Wittneben, Spectral efficient protocols for half-duplex fading relay channels. IEEE J. Selected Areas Commun. 25(2), 379-389 (2007)

15. G Farhadi, NC Beaulieu, On the ergodic capacity of wireless relaying systems over rayleigh fading channels. IEEE Trans. Wireless Commun. 7(11), 4462-4467 (2008)

16. K Azarian, H El Gamal, P Schniter, On the achievable diversity-multiplexing tradeoff in half-duplex cooperative channels. IEEE Trans. Inf. Theory. 51(12), 4152-4172 (2005)
17. MTO El Astal, AM Abu-Hudrouss, BP Salmon, JC Olivier, An adaptive transmission protocol for exploiting diversity and multiplexing gains in wireless relaying networks. EURASIP J. Wirel. Commun. Netw. 2015(1), $1-15$ (2015)

18. M Noori, M Ardakani, On the achievable rates of symmetric gaussian multi-way relay channels. EURASIP J. Wirel. Commun. Netw. 2013(1), 1-8 (2013)

19. SJ Kim, N Devroye, P Mitran, V Tarokh, Achievable rate regions and performance comparison of half duplex bi-directional relaying protocols. IEEE Trans. Inf. Theory. 57(10), 6405-6418 (2011)

20. S Ghasemi-Goojani, H Behroozi, Lattice-coded cooperation protocol for the half-duplex gaussian two-way relay channel. EURASIP J. Wirel. Commun. Netw. 2015(1), 1-18 (2015)

21. I Avram, N Aerts, M Moeneclaey, Low-complexity quantize-and-forward cooperative communication using two-way relaying. EURASIP J. Wirel. Commun. Netw. 2014(1), 1-10 (2014)

22. P Larsson, N Johansson, K-E Sunell, in Proceedings of IEEE Vehicular Technology Conference. Coded bi-directional relaying, (2006), pp. 851-855

23. K Song, B Ji, Y Huang, M Xiao, L Yang, Performance analysis of antenna selection in two-way relay networks. IEEE Trans. Signal Process. 63(10), 2520-2532 (2015)

24. TJ Oechtering, C Schnurr, I Bjelakovic, H Boche, Broadcast capacity region of two-phase bidirectional relaying. IEEE Trans. Inf. Theory. 54(1), 454-458 (2008)

25. R Ahlswede, N Cai, S-YR Li, RW Yeung, Network information flow. IEEE Trans. Inf. Theory. 46(4), 1204-1216 (2000)

26. TX Vu, P Duhamel, M Di Renzo, On the diversity of network-coded cooperation with decode-and-forward relay selection. IEEE Trans. Wireless Commun. 14(8), 4369-4378 (2015)

27. Y Liu, CW Sung, Network-coded retransmissions in wireless demodulate-and-forward relay channels. EURASIP J. Wirel. Commun. Netw. 2013(1), 1-14 (2013)

28. J Boutros, E Viterbo, Signal space diversity: a power- and bandwidth-efficient diversity technique for the Rayleigh fading channel. IEEE Trans. Inf. Theory. 44(4), 1453-1467 (1998)

29. Q Xie, J Song, K Peng, F Yang, Z Wang, Coded modulation with signal space diversity. IEEE Trans. Wireless Commun. 10(2), 660-669 (2011)

30. KN Pappi, ND Chatzidiamantis, GK Karagiannidis, Error performance of multidimensional lattice constellations - part II: evaluation over fading channels. IEEE Trans. Commun. 61(3), 1099-1110 (2013)

31. SA Ahmadzadeh, SA Motahari, AK Khandani, Signal space cooperative communication. IEEE Trans. Wireless Commun. 9(4), 1266-1271 (2010)

32. $\mathrm{T} L \mathrm{Lu}, \mathrm{J} \mathrm{Ge}, \mathrm{Y}$ Yang, $Y \mathrm{Gao}, \mathrm{BEP}$ analysis for DF cooperative systems combined with signal space diversity. IEEE Commun. Lett. 16(4), 486-489 (2012)

33. S Sohaib, S Zaheer, in Proceedings of IEEE Vehicular Technology Conference. Space time coded signal space diversity for multi-hop cooperative wireless communication, (2014), pp. 1-5

34. F Oggier, E Viterbo, Table of algebraic rotations. http://ecse.monash.edu. au/staff/eviterbo/rotations/rotations.html. Accessed 09 Dec 2015

35. F Oggier, Algebraic methods for channel coding. PhD thesis. EPFL (2005)

36. IEEE Standard for local metropolitan area networks (2004). Part 16: Air Interface for Fixed Broadband Wireless Access Systems. IEEE Std 802.16

37. M Abramowitz, IA Stegun, Handbook of Mathematical Functions with Formulas, Graphs, and Mathematical Tables, 10th edn. (National Bureau of Standards, U.S. Governement Printing Office, Washington, DC, 1972)

38. JG Proakis, DK Manolakis, Digital Signal Processing: Principles, Algorithms and Application, 4th edn. (Prentice Hall, Upper Saddle River, New Jersey, USA, 2006)

39. MK Simon, M-S Alouini, Digital Communications over Fading Channels, 2nd edn. (John Wiley \& Sons, New Jersey, USA, 2004)

40. IS Gradshteyn, IM Ryzhik, Table of Integrals, Series, and Products, 7th edn. (Elsevier Inc, San Diego, California, 2007)

41. KTourki, H-C Yang, M-S Alouini, Error-rate performance analysis of incremental decode-and-forward opportunistic relaying. IEEE Trans. Commun. 59(6), 1519-1524 (2011)

42. MR Bhatnagar, On the capacity of decode-and-forward relaying over rician fading channels. IEEE Commun. Lett. 17(6), 1100-1103 (2013) 